\title{
Theoretical Models of Superbursts on Accreting Neutron Stars
}

\section{Citation}

Cooper, Randall L., and Ramesh Narayan. 2005. “Theoretical Models of Superbursts on Accreting Neutron Stars." The Astrophysical Journal 629 (1): 422-37. https:// doi.org/10.1086/431273.

\section{Permanent link}

http://nrs.harvard.edu/urn-3:HUL.InstRepos:41384971

\section{Terms of Use}

This article was downloaded from Harvard University's DASH repository, and is made available under the terms and conditions applicable to Other Posted Material, as set forth at http:// nrs.harvard.edu/urn-3:HUL.InstRepos:dash.current.terms-of-use\#LAA

\section{Share Your Story}

The Harvard community has made this article openly available.

Please share how this access benefits you. Submit a story.

Accessibility 


\title{
Theoretical Models of Superbursts on Accreting Neutron Stars
}

\author{
Randall L. Cooper and Ramesh Narayan \\ Harvard-Smithsonian Center for Astrophysics, 60 Garden Street, Cambridge, MA 02138 \\ rcooper@cfa.harvard.edu, rnarayan@cfa.harvard.edu
}

\begin{abstract}
We carry out a general-relativistic global linear stability analysis of the amassed carbon fuel on the surface of an accreting neutron star to determine the conditions under which superbursts occur. We reproduce the general observational characteristics of superbursts, including burst fluences, recurrence times, and the absence of superbursts on stars with accretion rates $\dot{M}<0.1 \dot{M}_{\text {Edd }}$, where $\dot{M}_{\text {Edd }}$ denotes the Eddington limit. By comparing our results with observations, we are able to set constraints on neutron star parameters such as the stellar radius and neutrino cooling mechanism in the core. Specifically, we find that accreting neutron stars with ordered crusts and highly efficient neutrino emission in their cores (due to direct URCA or pionic reactions, for example) produce extremely energetic $\left(>10^{44}\right.$ ergs) superbursts which are inconsistent with observations, in agreement with previous investigations. Also, because of pycnonuclear burning of carbon, they do not have superbursts in the range of accretion rates at which superbursts are actually observed unless the crust is very impure. Stars with less efficient neutrino emission (due to modified URCA reactions, for example) produce bursts that agree better with observations. Stars with highly inefficient neutrino emission in their cores produce bursts that agree best with observations. Furthermore, we find that neutron stars with large radii $(R \sim 16 \mathrm{~km})$ produce very energetic superbursts that conflict with observations, even if the core neutrino emission mechanism is highly inefficient. Superburst characteristics are quite sensitive to several other parameters as well, most notably the composition of the accreted gas, concentration of carbon in the ignition region, and degree of crystallization of the crust. All systems that accrete primarily hydrogen and in which superbursts are observed show evidence of $\mathrm{H}$ - and He-burning delayed mixed bursts. We speculate that delayed mixed bursts provide sufficient amounts of carbon fuel for superbursts and are thus a prerequisite for having superbursts. We compare our global stability analysis to approximate one-zone criteria used by other authors and identify a particular set of approximations that give accurate results for most choices of parameters.
\end{abstract}


Subject headings: dense matter — nuclear reactions, nucleosynthesis, abundances — stars: neutron - X-rays: binaries - X-rays: bursts

\section{Introduction}

Superbursts are energetic thermonuclear flashes on the surfaces of accreting neutron stars thought to be caused by unstable carbon burning (Woosley \& Taam 1976; Taam \& Picklum 1978). They are similar to their hydrogen- and helium-burning Type I X-ray burst counterparts (which we refer to as "normal" bursts) in that they have thermal spectra, fast rises, and roughly exponential decays (see Strohmayer \& Bildsten 2004 for a review). Superbursts distinguish themselves, however, by their much larger fluences and longer recurrence times. Superbursts are a fairly new observational phenomenon: The first superburst was discovered in the system $4 \mathrm{U}$ 1735-444 by Cornelisse et al. (2000). Since then, eight more superbursts have been discovered in six other sources (see Kuulkers 2004 for a review). All nine superbursts had observed energies of $\sim 10^{42}$ ergs and were detected in systems with accretion rates between $10 \%$ and $30 \%$ of the Eddington limit. Recently, several more superburst candidates were observed in GX $17+2$, which accretes at a rate near the Eddington limit (in’t Zand et al. 2004a,b). Superburst recurrence times are not well constrained, though three have been observed within 4.7 years from the system 4U 1636-536 (Wijnands 2001; Strohmayer \& Markwardt 2002; Kuulkers et al. 2004).

Previous theoretical studies of superbursts (Brown \& Bildsten 1998; Cumming \& Bildsten 2001; Strohmayer \& Brown 2002) have been quite successful at modeling the general properties of superbursts. These models demonstrate that a large carbon-enriched layer on the surface of an accreting neutron star can indeed produce a thermonuclear burst due to unstable carbon burning if the accretion rate $\dot{M} \gtrsim 0.1 \dot{M}_{\text {Edd }}$. The resulting energetics and recurrence times of the thermonuclear flashes are roughly consistent with observations. However, these models employ an approximate criterion to estimate the column depth at which the unstable carbon ignition will occur. Specifically, they define ignition to occur at the depth at which the condition $\mathrm{d} \epsilon_{\text {nuc }} / \mathrm{d} T>\mathrm{d} \epsilon_{\text {cool }} / \mathrm{d} T$ is satisfied, where $\epsilon_{\text {nuc }}$ is the nuclear energy generation rate and $\epsilon_{\text {cool }}$ is an approximation to the cooling rate. Furthermore, these models integrate the stellar structure and time evolution equations only down to the superburst ignition region, applying physically-motivated but not necessarily self-consistent boundary conditions at both ends of their computational domains. However, the long characteristic accretion timescale for superbursts means that the thermal diffusion depth is quite large, often down to the core. Thus, to accurately model superbursts, one must solve for the thermal and hydrostatic profiles of the entire crust, well below the ignition region. Recently, 
Brown (2004) constructed an improved model that integrates the equations all the way to the core and solves for the inner temperature boundary condition using a method that is more self-consistent than previous studies. However, he uses the same approximate ignition criterion described above and assigns a fixed outer temperature boundary condition.

Some authors have attempted to go beyond these simple approximations in modeling normal Type I X-ray bursts. Fushiki \& Lamb (1987) were the first to discuss global perturbations of the steady-state configuration of the accreted layer. Their analysis was rather crude, however, since it assumed a constant temperature perturbation as a function of depth and did not treat perturbations below the accreted layer. Narayan \& Heyl (2003, hereafter Paper I) developed this approach much further by carrying out a full global linear stability analysis to determine the ignition conditions of normal Type I X-ray bursts, thereby putting thermonuclear burst theory on a more rigorous footing. However, their code has several limitations that, while relatively inconsequential for the study of normal bursts, are inadequate for the application to superbursts. Explicitly, the model omits carbon burning, is not fully general-relativistic, and is unable to integrate the stellar structure and time evolution equations below the depth at which neutron drip occurs. In the present investigation, we remove these limitations by significantly improving and expanding the burst model of Paper I. We then apply the new model to superbursts.

The motivation of this present study is to develop a mathematically more rigorous and self-consistent stability analysis to determine the physical conditions under which superbursts occur. Our goals are to compare and contrast our results to preceding theoretical work, determine the effects of a wide range of neutron star parameters upon superburst characteristics, and put the theory of superburst ignition on a more rigorous footing to enable quantitative comparisons with observations. Some of the results of this research were previously reported in Cooper \& Narayan (2004).

We begin the paper in $\S 2$ with a description of our numerical model, highlighting the additions to and improvements of the original model of Paper I. In $\S 3$, we discuss the thermal profile of the accreted layer and crust of the neutron star. In particular, we illustrate the effects on the thermal profile of varying several physical parameters such as the accretion rate, accreted gas composition, accreted layer composition, impurity concentration, degree of ion crystallization in the crust, and neutrino emission mechanism in the core. The consequences of these parameters upon the resulting superburst characteristics, notably the energetics and recurrence time, are significant, as we describe in $\S 4$. In $\S 5$ we compare our results with observations. We compare and contrast the results of our global linear stability analysis to those of the one-zone approximation in $\S 6$, and we conclude with a summary in $\S 7$. 


\section{The Model}

In this section, we outline the basic theoretical model, emphasizing the improvements over the original model described in Paper I. However, we will only briefly review the stability analysis procedure. The reader is encouraged to refer to Paper I for details.

\subsection{Governing Equations}

We assume that gas accretes spherically onto a compact object of gravitational mass $M$ and areal radius $R$ at a rate $\dot{M}$, where $\dot{M}$ is the rest mass accreted per unit time as measured by an observer at infinity. We consider all physical quantities to be functions of $\Sigma$, which we define as the rest mass of the accreted gas as measured from the top of the accreted layer divided by $4 \pi R^{2}$. Near the stellar surface, $\Sigma$ is properly interpreted as the column density. We use $\partial / \partial t$ and $\partial / \partial \Sigma$ to represent the Eulerian time and spatial derivatives, respectively, and $\mathrm{d} / \mathrm{d} t$ for the Lagrangian derivative following a parcel of gas:

$$
\frac{\mathrm{d}}{\mathrm{d} t}=\frac{\partial}{\partial t}+\frac{\dot{M}}{4 \pi R^{2}} e^{-\Phi / c^{2}} \frac{\partial}{\partial \Sigma} .
$$

Here $\Phi$ is the metric function (Misner et al. 1973), which reduces to the gravitational potential in the Newtonian limit. The term $e^{-\Phi / c^{2}}$ is equal to the redshift $1+z$ and relates time in the local frame to time at infinity. We include hydrogen, helium, and carbon burning for

the nuclear energy generation rates. Thus, we describe the composition of the gas by the hydrogen mass fraction $X$, helium fraction $Y$, CNO fraction $Z_{\mathrm{CNO}}$ and heavy element fraction $Z=1-X-Y-Z_{\mathrm{CNO}}$, where $Z$ refers to all metals other than CNO.

The stellar structure and time evolution of the accreting gas are governed by a set of nine partial differential equations (Thorne 1977; Brown 2000):

$$
\begin{gathered}
\frac{\partial r}{\partial \Sigma}=-\frac{R^{2}}{m_{b} n r^{2}}\left(1-\frac{2 G m}{r c^{2}}\right)^{1 / 2} \\
\frac{\partial m}{\partial \Sigma}=-\frac{4 \pi R^{2} \rho}{m_{b} n}\left(1-\frac{2 G m}{r c^{2}}\right)^{1 / 2} \\
\frac{\partial \Phi}{\partial \Sigma}=-\frac{G m R^{2}}{m_{b} n r^{4}}\left(1+\frac{4 \pi r^{3} P}{m c^{2}}\right)\left(1-\frac{2 G m}{r c^{2}}\right)^{-1 / 2} \\
\frac{\partial P}{\partial \Sigma}=\frac{G m R^{2} \rho}{m_{b} n r^{4}}\left(1+\frac{P}{\rho c^{2}}\right)\left(1+\frac{4 \pi r^{3} P}{m c^{2}}\right)\left(1-\frac{2 G m}{r c^{2}}\right)^{-1 / 2} \\
e^{-2 \Phi / c^{2}} \frac{\partial}{\partial \Sigma}\left(\frac{F r^{2}}{R^{2}} e^{2 \Phi / c^{2}}\right)=-T \frac{\mathrm{d} s}{\mathrm{~d} t}-\left(\epsilon_{\mathrm{H}}+\epsilon_{\mathrm{He}}+\epsilon_{\mathrm{C}}+\epsilon_{\mathrm{N}}-\epsilon_{\nu}\right)
\end{gathered}
$$




$$
\begin{gathered}
e^{-\Phi / c^{2}} \frac{\partial}{\partial \Sigma}\left(T e^{\Phi / c^{2}}\right)=\frac{3 R^{2} F \rho \kappa}{16 \sigma m_{b} n T^{3} r^{2}}, \\
\frac{\mathrm{d} X}{\mathrm{~d} t}=-\frac{\epsilon_{\mathrm{H}}}{E_{\mathrm{H}}^{*}}, \\
\frac{\mathrm{d} Y}{\mathrm{~d} t}=\frac{\epsilon_{\mathrm{H}}}{E_{\mathrm{H}}^{*}}-\frac{\epsilon_{\mathrm{He}}}{E_{\mathrm{He}}^{*}}, \\
\frac{\mathrm{d} Z_{\mathrm{CNO}}}{\mathrm{d} t}=\frac{\epsilon_{\mathrm{He}}}{E_{\mathrm{He}}^{*}}-\frac{\epsilon_{\mathrm{C}}}{E_{\mathrm{C}}^{*}} .
\end{gathered}
$$

Note that equation (5) is the Tolman-Oppenheimer-Volkoff equation of hydrostatic equilibrium. In these equations $m$ is the interior gravitational mass, $\rho$ is the mass density (such that $\rho c^{2}$ is the energy density), $m_{b}$ is the mass of one baryon, $n$ is the baryon number density, $r$ is the areal (Schwarzschild) radius, $P$ is the pressure, $F$ is the energy flux, $T$ is the temperature, $s$ is the entropy per unit mass, $\epsilon_{\mathrm{H}}, \epsilon_{\mathrm{He}}, \epsilon_{\mathrm{C}}$, and $\epsilon_{\mathrm{N}}$ are the energy generation rates due to hydrogen burning, helium burning, carbon burning, and deep crustal heating, $\epsilon_{\nu}$ is the energy loss rate due to neutrino emission, $\kappa$ is the opacity, and $E_{\mathrm{H}}^{*}, E_{\mathrm{He}}^{*}$, and $E_{\mathrm{C}}^{*}$ are the total nuclear energies released per unit mass of hydrogen, helium, and carbon burned, respectively.

Note that in the post-Newtonian stellar structure equations, the partial derivatives of the physical parameters are taken with respect to the Eulerian variable $\Sigma$, while the derivatives should be taken with respect to a Lagrangian variable. However, we solve the equations in quasi-steady state. Specifically, to find the equilibrium configuration, we set $\partial / \partial t=0$ and solve the following set of ordinary differential equations

$$
\begin{gathered}
\frac{\mathrm{d} r}{\mathrm{~d} \Sigma}=-\frac{R^{2}}{m_{b} n r^{2}}\left(1-\frac{2 G m}{r c^{2}}\right)^{1 / 2}, \\
\frac{\mathrm{d} m}{\mathrm{~d} \Sigma}=-\frac{4 \pi R^{2} \rho}{m_{b} n}\left(1-\frac{2 G m}{r c^{2}}\right)^{1 / 2}, \\
\frac{\mathrm{d} \Phi}{\mathrm{d} \Sigma}=-\frac{G m R^{2}}{m_{b} n r^{4}}\left(1+\frac{4 \pi r^{3} P}{m c^{2}}\right)\left(1-\frac{2 G m}{r c^{2}}\right)^{-1 / 2}, \\
\frac{\mathrm{d} P}{\mathrm{~d} \Sigma}=\frac{G m R^{2} \rho}{m_{b} n r^{4}}\left(1+\frac{P}{\rho c^{2}}\right)\left(1+\frac{4 \pi r^{3} P}{m c^{2}}\right)\left(1-\frac{2 G m}{r c^{2}}\right)^{-1 / 2}, \\
e^{-2 \Phi / c^{2}} \frac{\mathrm{d}}{\mathrm{d} \Sigma}\left(\frac{F r^{2}}{R^{2}} e^{2 \Phi / c^{2}}\right)=-T \frac{\dot{M}}{4 \pi R^{2}} e^{-\Phi / c^{2}} \frac{\mathrm{d} s}{\mathrm{~d} \Sigma}-\left(\epsilon_{\mathrm{H}}+\epsilon_{\mathrm{He}}+\epsilon_{\mathrm{C}}+\epsilon_{\mathrm{N}}-\epsilon_{\nu}\right), \\
e^{-\Phi / c^{2}} \frac{\mathrm{d}}{\mathrm{d} \Sigma}\left(T e^{\Phi / c^{2}}\right)=\frac{3 R^{2} F \rho \kappa}{16 \sigma m_{b} n T^{3} r^{2}}, \\
\frac{\mathrm{d} X}{\mathrm{~d} \Sigma}=-\frac{4 \pi R^{2}}{\dot{M}} e^{\Phi / c^{2}} \frac{\epsilon_{\mathrm{H}}}{E_{\mathrm{H}}^{*}},
\end{gathered}
$$




$$
\begin{gathered}
\frac{\mathrm{d} Y}{\mathrm{~d} \Sigma}=\frac{4 \pi R^{2}}{\dot{M}} e^{\Phi / c^{2}}\left(\frac{\epsilon_{\mathrm{H}}}{E_{\mathrm{H}}^{*}}-\frac{\epsilon_{\mathrm{He}}}{E_{\mathrm{He}}^{*}}\right), \\
\frac{\mathrm{d} Z_{\mathrm{CNO}}}{\mathrm{d} \Sigma}=\frac{4 \pi R^{2}}{\dot{M}} e^{\Phi / c^{2}}\left(\frac{\epsilon_{\mathrm{He}}}{E_{\mathrm{He}}^{*}}-\frac{\epsilon_{\mathrm{C}}}{E_{\mathrm{C}}^{*}}\right) .
\end{gathered}
$$

Clearly, since the compact object continuously accretes matter from its companion, the system is never precisely in equilibrium. However, the accretion timescale is longer than that of most of the relevant physical processes, so the steady-state approximation is acceptable. In this case, $\Sigma$ becomes a good Lagrangian variable, and so the use of $\Sigma$ as our independent variable is justified.

Stable and/or unstable hydrogen and helium burning produces most of the carbon that ultimately triggers a superburst. As of this writing, each system with an observed superburst also undergoes normal Type I X-ray bursts (Kuulkers 2004; in't Zand et al. 2004a). The carbon yield resulting from both stable helium burning and unstable burning during normal bursts is uncertain (see $\S 4$ ). To account for this uncertainty, we introduce a free parameter $C_{\mathrm{f}}$, the fraction of hydrogen and helium that ultimately burns to carbon. Equation (19) thus becomes

$$
\frac{\mathrm{d} Z_{\mathrm{CNO}}}{\mathrm{d} \Sigma}=\frac{4 \pi R^{2}}{\dot{M}} e^{\Phi / c^{2}}\left(C_{\mathrm{f}} \frac{\epsilon_{\mathrm{He}}}{E_{\mathrm{He}}^{*}}-\frac{\epsilon_{\mathrm{C}}}{E_{\mathrm{C}}^{*}}\right),
$$

so at the base of the accreted layer, $Z_{\mathrm{CNO}} \approx C_{\mathrm{f}}$. Clearly this is an approximation. However, it enables us to model the composition of the accreted layer as well as possible. Specifically, we can model the composition of both the hydrogen- and helium-rich upper region of the outer crust and the carbon-rich lower region to high accuracy. Additionally, the layer in which the hydrogen and helium burn to carbon is most likely very narrow in the column density $\Sigma$, so this transition region is inconsequential to the thermal and hydrostatic profiles of the outer crust.

\subsection{Boundary Conditions}

The solution to the set of nine coupled partial differential equations (11-19) requires nine separate boundary conditions. Eight are applied at the photosphere (where the optical depth $\tau_{\text {out }}=2 / 3$ ) and one is applied at the crust-core interface.

The outer boundary conditions for equations (11-13) are $r=R, m=M$, and $\Phi=$ $\left(c^{2} / 2\right) \ln \left(1-2 G M / R c^{2}\right)$. The outer boundary conditions for the hydrogen, helium, and CNO mass fraction evolution equations (17-19) are given by the composition of the accreting gas, such that $X=X_{\text {out }}, Y=Y_{\text {out }}$, and $Z_{\mathrm{CNO}}=Z_{\mathrm{CNO} \text {,out }}$, respectively. The value for $\Sigma$ at the photosphere, $\Sigma_{\text {out }}$, is obtained approximately by taking the opacity at the photosphere to be 
given by electron scattering. Thus, $\Sigma_{\text {out }}=\tau_{\text {out }} /\left(0.2\left(1+X_{\text {out }}\right)\right) \mathrm{g} \mathrm{cm}^{-2}$. The outer boundary condition for equation (14) is then given by

$$
P_{\text {out }}=\Sigma_{\text {out }} \frac{G M}{R^{2}}\left(1-\frac{2 G M}{R c^{2}}\right)^{-1 / 2} .
$$

The method we use to determine the outer boundary condition for equation (15), the outward flux at the stellar surface $F_{\text {out }}$, is explained in Paper I. To summarize, we assume a given value of $F_{\text {out }}$ which, when added to the gravitational energy flux due to accretion, also defines a surface temperature $T_{\text {out }}$. We then integrate the differential equations and compare the resulting temperature at the bottom, the crust-core interface, to the required temperature inner boundary condition. We adjust $F_{\text {out }}$ and repeat the integration until the temperature boundary condition at the bottom is satisfied to high accuracy. What makes our new method superior to that of Paper I is that we now integrate all the way to the stellar core $\left(\rho_{0} \approx 2 \times 10^{14} \mathrm{~g} \mathrm{~cm}^{-3}\right.$, where $\rho_{0}$ denotes the rest mass density). Previously, we integrated only a couple of diffusion depths into the star, but we were unable to integrate past the neutron drip point $\left(\rho_{0} \approx 4 \times 10^{11} \mathrm{~g} \mathrm{~cm}^{-3}\right)$. The long recurrence times of superbursts make the old method inadequate. For many calculations, the thermal diffusion depth is deeper than the crust-core interface, so integration to the core is necessary.

For a given calculation, we employ one of two methods to determine the temperature inner boundary condition for equation (16). In our first method, we assume that we know the rate of neutrino emission from the core; we use two prescriptions for this, either modified URCA reactions (Friman \& Maxwell 1979; Yakovlev \& Levenfish 1995) or pionic reactions (Maxwell et al. 1977). Integrating to the crust-core interface gives values for the proper temperature $T$, areal radius $r$, interior gravitational mass $m$, and energy flux $F$. The energy flux, which is directed inward at the interface $(F<0)$, must be balanced by the neutrino cooling of the core. Using the neutrino luminosities $L_{\nu}(m, T)$ from Shapiro \& Teukolsky (1983), we determine the core temperature $T_{\text {core }}$ via the equation

$$
L_{\nu}\left(m, T_{\text {core }}\right)=-4 \pi r^{2} F .
$$

We then compare $T_{\text {core }}$ to $T$ and iterate until they match. Note that we do not modify the formulae to account for the volume of the core. Since we do not know the equation of state of the core, we cannot solve for the core's proper volume. However, since the formulae are estimates, and since the luminosity is a very strong function of the temperature $\left(L_{\nu}^{\text {mURCA }} \propto T^{8}\right.$ and $\left.L_{\nu}^{\pi} \propto T^{6}\right)$, this approximation is innocuous. In our second method, we simply assign a value for the core temperature $T_{\text {core }}$ and iterate until $T=T_{\text {core }}$ at the crust-core interface. 


\subsection{Auxiliary Conditions}

\subsubsection{Equation of State}

We assume photons, electrons, ions, and free neutrons supply the pressure. To calculate the photon and electron contributions to the pressure, we follow the same procedure as in Paper I, while for the ions and free neutrons, we follow the prescriptions of Brown (2000). The phase of the ions, whether solid or liquid, is determined by the dimensionless coupling parameter

$$
\Gamma=\frac{(Z e)^{2}}{k_{\mathrm{B}} T}\left(\frac{4 \pi}{3} n_{I}\right)^{1 / 3}
$$

where $n_{I}$ is the ion number density. In the outermost layers of the crust, where $\Gamma<1$, we use the free energy fits of Chabrier \& Potekhin (1998) for the ion equation of state. For $\Gamma>1$ we use the analytical fits of Farouki \& Hamaguchi (1993) for the Helmholtz free energy to calculate the ion pressure. We assume the ions are in the liquid phase when $1<\Gamma<173$ and the solid phase when $\Gamma>173$. The analytical fits are valid for a one-component plasma. However, in the accreted layer more than one species is usually present at a given density. In this case we approximate the multi-component mixture as a one-component plasma by making the substitutions $Z \rightarrow\langle Z\rangle$ and $n_{I} \rightarrow n / \mu_{n u c}$, where $\mu_{n u c}$ is the mean molecular weight per nucleus. Below the accreted layer, we assume only one species is present at a given density. We calculate the free neutron pressure from the compressible liquid-drop nuclear model of Mackie \& Baym (1977). For $\rho_{0}>1.3 \times 10^{13} \mathrm{~g} \mathrm{~cm}^{-3}$, we use the equation of state from Negele \& Vautherin (1973).

The photon entropy formula is the same as that in Paper I. We calculate the Coulomb portion of the ion entropy from the free energy fits of Chabrier (1993) and the ideal portion from the fits of Farouki \& Hamaguchi (1993). The treatment of the electron and free neutron entropies is more difficult. The compressional heating terms in equation (15) are unlikely to affect the thermal profile calculation significantly (Brown \& Bildsten 1998) and therefore are often omitted in other investigations. However, we attempt to make a reasonable approximation to the compressional heating terms relevant to our work. We consider three entropy "regimes": ideal, nonrelativistic degenerate, and extremely relativistic degenerate. Define $\Theta=m_{\mathrm{p}} k_{\mathrm{B}} T /\left(\hbar^{2} n^{2 / 3}\right)$ and $x=\left(\hbar / m_{\mathrm{p}} c\right)\left(3 \pi^{2} n\right)^{1 / 3}$, the relativity parameter (Shapiro \& Teukolsky 1983), where $m_{\mathrm{p}}$ is the particle mass, $k_{\mathrm{B}}$ is Boltzmann's constant, and $n$ is the particle number density. Then the expressions for the entropy of a species in each of the three regimes are (Landau \& Lifshitz 1969)

$$
\frac{s_{\text {ideal }}}{k_{\mathrm{B}}}=\frac{3}{2} \ln (\Theta)+\ln \left(\frac{2}{(2 \pi)^{3 / 2}}\right)+\frac{5}{2},
$$




$$
\begin{gathered}
\frac{s_{\mathrm{NRD}}}{k_{\mathrm{B}}}=\left(\frac{\pi}{3}\right)^{2 / 3} \Theta=\frac{\pi^{2} k_{\mathrm{B}} T}{m_{\mathrm{p}} c^{2} x^{2}}, \\
\frac{s_{\mathrm{ERD}}}{k_{\mathrm{B}}}=\frac{\pi^{2} k_{\mathrm{B}} T}{m_{\mathrm{p}} c^{2} x} .
\end{gathered}
$$

Note that $\Theta \gg 1$ for an ideal gas and $\Theta \ll 1$ for a degenerate gas. Similarly, $x \gg 1$ for a relativistic gas and $x \ll 1$ for a nonrelativistic gas. The question is, then, how does one decide where to make the transitions between the three regimes? It is evident from equation (15) that only the entropy derivatives, not the entropy values, are important. Therefore, we choose the transition values of $\Theta$ and $x$ in such as way as to make the partial derivatives of the entropies with respect to the variable $\Theta$ or $x$ continuous functions of that variable. First, we decide whether the species is ideal or degenerate. Thus, for a quantity $q$, we first find the transition value $\Theta_{\text {trans }}$ such that $\partial s_{\text {ideal }} / \partial q=\partial s_{\mathrm{NRD}} / \partial q$ at $\Theta=\Theta_{\text {trans. }}$. For all quantities $q$, $\Theta_{\text {trans }}=1.455$. If the species is degenerate, we then decide whether it is nonrelativistic or extremely relativistic. We then repeat the same procedure with $\Theta \rightarrow x . x_{\text {trans }}=0.5,1$, or 2 , depending on the specific quantity $q$.

\subsubsection{Nuclear Energy Generation Rates}

The hydrogen and helium nuclear energy generation rates are identical to those in Paper I with the exceptions that the ${ }^{13} \mathrm{~N}(p, \gamma){ }^{14} \mathrm{O}$ and ${ }^{14} \mathrm{~N}(p, \gamma){ }^{15} \mathrm{O}$ rates for $\epsilon_{\mathrm{H}}$ are updated to those of Caughlan \& Fowler (1988).

Carbon burns through several different energetically possible reaction channels (Clayton 1983). For simplicity, we assume that the dominant reaction is ${ }^{12} \mathrm{C}\left({ }^{12} \mathrm{C}, \gamma\right){ }^{24} \mathrm{Mg}$, so $E_{\mathrm{C}}^{*}=$ $5.6 \times 10^{17} \mathrm{erg} \mathrm{g}^{-1}$ (Cumming \& Bildsten 2001). For $\epsilon_{\mathrm{C}}$, we use both the reaction rates and electron screening enhancement factors of Kitamura (2000). We interpolate the logarithms of the fluid and solid phase reaction rates in the same way that Kitamura (2000) interpolates the thermonuclear and pycnonuclear enhancement factors. This makes the energy generation rate a smooth function of both density and temperature. See Figure 1 for a plot of $\epsilon_{\mathrm{C}}$ as a function of $\rho_{0}$. Note that we use the approximate criterion of Kitamura (2000) for the solidification of an electron-screened binary ionic mixture. Previous authors (Brown \& Bildsten 1998; Cumming \& Bildsten 2001; Strohmayer \& Brown 2002; Brown 2004) have used the reaction rate of Caughlan \& Fowler (1988) with the enhancement factor of Ogata et al. (1993). This energy generation rate is valid only in the liquid phase, where the burning is considered strictly thermonuclear. In contrast, our energy generation rate is valid in both the thermonuclear regime, where the crust is fluid, and the pycnonuclear regime, where the crust is solid. The two energy generation rates agree to sufficient precision in 
the thermonuclear regime. In the pycnonuclear regime, the nuclear energy generation rate is essentially temperature-independent. Including this regime in the model can sometimes be important, especially in neutron stars with efficient core cooling mechanisms or heavyelement oceans.

\subsection{Deep Crustal Heating and Neutrino Emission}

For $6 \times 10^{11} \mathrm{~g} \mathrm{~cm}^{-3} \lesssim \rho_{0} \lesssim 3 \times 10^{13} \mathrm{~g} \mathrm{~cm}^{-3}$, electron captures, neutron emissions, and pycnonuclear reactions release $\approx 1 \mathrm{MeV}$ per baryon over a timescale inversely proportional to the accretion rate (Haensel \& Zdunik 1990a,b; Brown et al. 1998). For $\epsilon_{\mathrm{N}}$, the energy generation rate due to this deep crustal heating, we use the formula of Brown (2000).

We include pair, photo-, plasma, and bremsstrahlung neutrino emission processes for the neutrino energy loss rate $\epsilon_{\nu}$. For the pair, photo-, and plasma processes we use the analytical fits of Itoh et al. (1996), and for the bremsstrahlung emission rate we use the work of Haensel et al. (1996) when the ions are in the liquid phase and Yakovlev \& Kaminker (1996) when the ions are in the solid phase.

\section{Thermal Structure of the Accreted Layer and Crust}

Superburst characteristics depend sensitively upon the thermal structure of the neutron star crust. In this section, we identify some of the key parameters that affect the thermal profile.

\subsection{Accretion Rate}

In its journey from the binary companion to the neutron star core, a parcel of matter releases a tremendous amount of energy, and the rate of this energy release is directly proportional to the rate at which matter falls onto the stellar surface. Thus, it is not surprising that the thermal profile of an accreting neutron star is very sensitive to the accretion rate. To varying degrees of importance, the accretion rate affects the thermal profile in four different ways.

Most of the energy released by an infalling parcel of accreted material is from the gravitational energy released when the matter impacts the stellar surface. Most of this energy is radiated outward. Nevertheless, it determines the temperature at the stellar surface and 
thereby sets a boundary condition. The calculation is rather insensitive to this temperature, however, for the thermal profile near the surface approaches a radiative-zero solution. As accretion continues, nuclear fuel accumulates and eventually burns either stably or unstably. The time-averaged rate of nuclear energy generation is proportional to the accretion rate. Although the nuclear energy per gram of accreted material released via fusion is roughly forty times less than that released from gravitational energy, the nuclear energy is generated well below the stellar surface. Thus the nuclear energy generation can have a significant effect upon the thermal profile of the crust, in particular the superburst ignition region (see also §3.2). Additionally, continuous accretion causes both compressional heating throughout the crust and deep crustal heating via non-equilibrium reactions. The compressional heating is rather small compared to other sources, and it is therefore often neglected in other studies. The deep crustal heating is roughly five times less than that from hydrogen and helium burning, but it can have a non-negligible effect upon the thermal profile of the crust, especially if the conductive opacity of the inner crust is large.

We plot the temperature and flux profiles for two neutron stars accreting at different rates in Figure 2. The energy flux is normalized by the maximum nuclear burning energy flux available in the accreting gas:

$$
F_{\text {nuc }}=\frac{\dot{M}}{4 \pi R^{2}}\left(1-\frac{2 G M}{R c^{2}}\right)^{-1 / 2}\left[X_{\text {out }} E_{\mathrm{H}}^{*}+\left(X_{\text {out }}+Y_{\text {out }}\right) E_{\mathrm{He}}^{*}+\left(X_{\text {out }}+Y_{\text {out }}+Z_{\mathrm{CNO}, \text { out }}\right) E_{\mathrm{C}}^{*}\right] .
$$

The parameter $l_{\text {acc }}=\dot{M} / \dot{M}_{\text {Edd }}$ is the accretion rate normalized to the Eddington limit, where $\dot{M}_{\mathrm{Edd}}=4 \pi G M(1+z) / c z \kappa_{\mathrm{es}}$, with $\kappa_{\mathrm{es}}=0.4 \mathrm{~cm}^{2} \mathrm{~g}^{-1}$. The temperature outer boundary condition, which is shown at the left end of the left panel, is determined by the rate of gravitational energy liberated at the surface. Changes in the slope of the temperature profile are associated with localized energy sources, and they are reflected by rapid changes in the flux. Thus, hydrogen and helium burning causes the peak in the thermal profile and the large change of flux at a column depth $\Sigma \approx 10^{8} \mathrm{~g} \mathrm{~cm}^{-2}$. A small amount of carbon burning occurs at $\Sigma \sim 10^{12}-10^{13} \mathrm{~g} \mathrm{~cm}^{-2}$. Deep crustal heating occurs for column depths $10^{15}$ $\mathrm{g} \mathrm{cm}^{-2} \lesssim \Sigma \lesssim 10^{17} \mathrm{~g} \mathrm{~cm}^{-2}$. The flux profile shows that most of the energy generated by deep crustal heating is directed inward, in agreement with Brown (2000).

\subsection{Energy Generated from Hydrogen and Helium Burning}

The burning of hydrogen and helium near the surface of an accreting neutron star releases a substantial amount of energy within the star. Consequently, the thermal profile of the outer crust, including the superburst ignition region, is rather sensitive to the magnitude and physical location of this energy generation. To account for this, previous authors 
set the temperature at a given column depth to coincide with estimates from investigations of hydrogen and helium ignition. However, the thermal profile in this region is a sensitive function of many variables, including the mass accretion rate (e.g., compare the two models in Fig. 2), stellar radius, and composition of the accreted gas. Since we include both hydrogen and helium energy generation rates in our energy conservation equation, we make no assumptions regarding the temperature at a given depth in the accreted layer. Thus, we are able to determine the thermal profile of the outer crust self-consistently. Not only does this improve the accuracy of our calculation, but it also gives us the freedom to vary physical parameters such as the gas composition and stellar radius self-consistently.

As noted earlier, all of the systems in which astronomers have observed superbursts exhibit normal Type I X-ray bursts as well. To do a rigorous calculation of the thermal profile of the outer crust, one would need to conduct a fully time-dependent calculation of many successive normal bursts, which is beyond the scope of this study. Our calculation is quasistatic, so the composition and thermal profile of the crust in the the normal burst ignition region is essentially computed via stable (though rapid) hydrogen and helium burning. Since the timescale over which normal bursts occur (hours to days) is much shorter than the timescale over which superbursts occur (years to possibly decades), any effects that hydrogen and helium burning have on the thermal profile of the superburst ignition region will be due to the time-averaged hydrogen and helium nuclear energy generation rate. The time-averaged energy generation rate is the same regardless of the manner in which the fuel is burned. Therefore, our method should be sufficiently accurate for our purposes.

To demonstrate the importance of hydrogen and helium burning on the thermal profile of the outer crust, we plot in Figure 3 the temperature as a function of column density for two systems with different accreted gas compositions. Hydrogen burning releases much more energy per gram of accreted fuel than helium burning. Therefore, the maximum temperature achieved in the outer crust is in general proportional to the mass fraction of hydrogen of the accreted gas. This can have a significant effect on superburst characteristics (see $§ 4.1$ ). The inner crust $\left(\Sigma \gtrsim 10^{15} \mathrm{~g} \mathrm{~cm}^{-2}\right.$ ), however, is rather insulated from the hydrogen and helium burning region, so the thermal profile of the inner crust is rather insensitive to the hydrogen and helium burning near the surface.

\subsection{Ash Composition from Hydrogen and Helium Burning}

The heavy element composition of the accreted layer where superbursts are triggered is uncertain. Especially for mixed hydrogen/helium accretors, the primary cause of this uncertainty is due to the rp-process (Wallace \& Woosley 1981), whose ashes are probably a 
mix of elements beyond the iron peak (Schatz et al. 2001). Type I X-ray burst models of Woosley et al. (2004) produce nuclei with an average atomic weight $\langle A\rangle \approx 64$. Therefore, we choose two representative metals as our heavy elements: (i) ${ }_{26}^{56} \mathrm{Fe}$, and (ii) following Cumming

\& Bildsten (2001), ${ }_{44}^{104} \mathrm{Ru}$. These choices likely bracket the true average atomic weight and electric charge of the ashes. We assume that the nuclear composition below the accreted layer is that of Haensel \& Zdunik (1990a,b) for both cases. Haensel \& Zdunik (2003) later studied the evolution of heavy $(\langle A\rangle \approx 100)$ rp-process ashes. However, Schatz et al. (2003a) showed that the high temperatures reached during a superburst might induce photodisintegration reactions in the heavy ashes, converting them to iron group elements. Thus, we presume that the outer crust below the accreted layer consists chiefly of iron group elements, with possible impurities.

We find that, contrary to the study of Cumming \& Bildsten (2001), the composition of the ashes from hydrogen and helium has a negligible effect on the thermal profile of the crust. This is consistent with the results of Brown (2004). See Figure 4 for a plot of the thermal profile for neutron star crusts with different ash compositions.

\subsection{Core Temperature}

The thermal profile of both the inner crust and the outer crust below the hydrogen/helium burning region is very sensitive to the temperature at the crust-core interface. See Figure 5 for a plot of the thermal profiles of neutron stars with different core temperatures. In particular, note the slopes of the profiles beyond the hydrogen/helium burning region, at $\Sigma \approx 10^{8} \mathrm{~g} \mathrm{~cm}^{-2}$. Except for neutron stars with very hot cores $\left(T \gtrsim 4 \times 10^{8} \mathrm{~K}\right)$, the temperature gradient $\partial T / \partial \Sigma$ in this region is negative (i.e. $\partial T / \partial r$ is positive), which implies that the net energy flux is negative. This is clearly illustrated in the energy flux profiles in Figure 5. Previous studies of thermonuclear burst ignition have often used the result of Brown (2000) for the inner flux boundary condition. He found that approximately $10 \%$ of the energy generated in the crust through deep crustal heating flows outward. In Figure 5 this corresponds to $F / F_{\text {nuc }} \approx 0.02$. For comparison, the energy flux in Figure 5 just below the column depth at which the superburst is triggered ranges from $F / F_{\text {nuc }} \approx+0.05$ at $\Sigma \approx 10^{11.7}$ $\mathrm{g} \mathrm{cm}^{-2}$ for the hottest thermal profile to $F / F_{\text {nuc }} \approx-0.06$ at $\Sigma \approx 10^{14.0} \mathrm{~g} \mathrm{~cm}^{-2}$ for the coldest. Brown's results, which are accurate for the particular physical scenario he studied and which we are able to reproduce, are clearly not applicable for all scenarios. As we have shown, the thermal profile of an accreting neutron star is sensitive to many parameters, including the accretion rate, composition of the accreted gas, and core temperature. Therefore, one must use caution when implementing flux or temperature boundary conditions. In our opinion, it 
is preferable to do self-consistent calculations, as in this work.

In recent work, Brown (2004) has used an approximate "outer" boundary condition on the temperature: $T=2.5 \times 10^{8} \mathrm{~K}$ at $\Sigma=10^{9} \mathrm{~g} \mathrm{~cm}^{-2}$. A quick look at Figures 2, 3, and 5 here shows that the temperature varies considerably at this depth, depending on various parameters. Also, $T$ is typically greater than $2.5 \times 10^{8} \mathrm{~K}$ at this depth.

\subsection{Ion Impurities in the Crust}

Electron-impurity scattering (Itoh \& Kohyama 1993) and electron-phonon scattering (Baiko \& Yakovlev 1995) determine the thermal conductivity of the solidified inner crust of an accreting neutron star. Ion impurities embedded in a crystalline lattice cause deviations in an otherwise periodic potential. Electron scattering off of these deviations reduces the thermal conductivity of the lattice and consequently raises the temperature of the inner crust. Unlike electron-phonon scattering, which is due to thermal oscillations of ions and is therefore temperature-dependent, electron-impurity scattering depends primarily on the lattice composition and thus the corresponding collision frequency is essentially temperatureindependent. Therefore, impurities can have a significant effect on the thermal conductivity of the crust, especially at low temperatures (Itoh \& Kohyama 1993; Ashcroft \& Mermin 1976).

The contribution of impurities to the thermal conductivity of the crust is parametrized by the impurity parameter (Itoh \& Kohyama 1993; Brown 2000)

$$
Q \equiv \frac{1}{n_{I}} \sum_{j} n_{j}\left(Z_{j}-\langle Z\rangle\right)^{2},
$$

where $n_{j}$ and $Z_{j}$ are the number density and charge of the $j$ th ion species, $n_{I}=\sum_{j} n_{j}$ is the total ion number density, and $\langle Z\rangle=n_{I}^{-1} \sum_{j} n_{j} Z_{j}$ is the mean charge. Sources of impurities in the crust include electron captures and pycnonuclear reactions (Haensel \& Zdunik 1990a,b), but the main source of impurities is most likely the mixture of superburst and/or rp-process ashes at the top of the substrate. Early studies of the composition of rpprocess ashes implied that impurity scattering should be significant, with $Q \sim 100$ (Schatz et al. 1999). Subsequent calculations by Woosley et al. (2004) and Koike et al. (2004) showed that rp-process ashes consist chiefly of iron-peak nuclei, resulting in a lower $Q$. Schatz et al. (2003a) found that $Q \approx 5.2$ after a superburst is triggered. In our model, we calculate $Q$ self-consistently within the accreted layer, and we adopt $Q=5.2$ as our fiducial impurity parameter value in the substrate below the accreted layer. Figure 6 illustrates the effects of electron-impurity scattering on the thermal profile of an accreting neutron star. 


\subsection{Ion Crystallization in the Crust}

When calculating the conductive opacity in the inner crust of the neutron star, we usually assume that the ions form an ordered crystal lattice when the dimensionless coupling parameter $\Gamma>173$ (eq. 23). In this case, the conductive opacity is usually dominated by electron-phonon scattering due to oscillations of the ions in the lattice (Potekhin et al. 1999). However, previous studies of the nuclear structure of the inner crust suggest that some fraction of the inner crust may in fact be disordered (Magierski \& Heenen 2002; Magierski \& Bulgac 2004). Following Brown (2004), we investigate the thermal profile of a completely disordered neutron star crust. We use the thermal conductivity expression of Itoh \& Kohyama (1993) and set the structure factor $\langle S\rangle$ to unity. Additionally, we set the squared impurity charge $\left\langle(\Delta Z)^{2}\right\rangle=\langle Z\rangle^{2}$. This essentially sets a lower limit on the thermal conductivity of the crust (E. Brown, private communication). The thermal profiles of the crusts for neutron stars with cores that emit neutrinos via either modified URCA reactions or pionic reactions are shown in Figure 7. In contrast to neutron stars with crystalline crusts (Fig. 5), the thermal profiles now are quite insensitive to the nature of the core neutrino cooling mechanism.

\section{Results}

\subsection{Composition of the Accreted Gas}

For systems that have exhibited a superburst and for which the composition of the accreted gas can be reasonably estimated, all but one accrete a mixture of hydrogen and helium, with hydrogen being the most abundant species by mass (Kuulkers 2004). The system 4U 1820-30 (Strohmayer 2000; Strohmayer \& Brown 2002) is the exception. Several studies (Fedorova \& Ergma 1989; Podsiadlowski et al. 2002; Cumming 2003) imply that the compact star in this system accretes a helium-rich mixture with a small hydrogen mass fraction $X \sim 0.1$. Observationally, the superburst from $4 \mathrm{U} 1820-30$ is distinct, with a larger fluence, luminosity, and peak temperature than every other superburst observed thus far (Kuulkers et al. 2002b; Kuulkers 2004). Therefore, to determine the effects of accreted gas composition on superbursts, we choose two different elemental abundances: (i) "mixed hydrogen/helium", for which the mass fractions of the accreted gas are $X=0.7, Y=0.28$, and $Z_{\mathrm{CNO}}=0.016$, and (ii) "helium", for which $X=0.1, Y=0.88$, and $Z_{\mathrm{CNO}}=0.016$.

Figure 8 shows the superburst energies and recurrence times as a function of accretion rate, as calculated by our model. All other parameters being equal, helium accretors require a larger column density of accreted gas before a superburst is triggered. Therefore, their 
superbursts are more energetic and have longer recurrence times, in agreement with the observations of $4 \mathrm{U}$ 1820-30. Helium burning releases less energy per gram than hydrogen burning by approximately one order of magnitude. Consequently, for a given column density, the temperature at the base of the layer is lower (see Fig. 3), so a larger column of fuel must accumulate before a superburst can occur. This disparity is greater at high accretion rates, for which a lower column density is required for an instability. Less matter exists between the hydrogen/helium burning region and the base of the layer, so the base is less insulated from the burning region and therefore more sensitive to the energy generated there.

As we have shown, the composition of the accreted gas affects superburst characteristics by its effect upon the thermal profile of the outer crust where superbursts are triggered. However, the composition may have an even greater influence on superburst characteristics through the carbon yield resulting from both the stable and unstable burning of the gas. Unfortunately, we cannot investigate this aspect of the problem with our model since we do not solve for the carbon yield self-consistently, but set it through the parameter $C_{\mathrm{f}}$. We assume the fiducial value $C_{\mathrm{f}}=0.3$ for all calculations unless notified otherwise.

\subsection{Composition of the Ashes in the Accreted Layer}

The composition of the accreted layer where superbursts are triggered is uncertain. In particular, the mass fraction of carbon produced via stable and unstable hydrogen and helium burning is unknown, but it most likely has to be $\gtrsim 10 \%$ for mixed hydrogen/helium accretors (Cumming \& Bildsten 2001) and $\gtrsim 30 \%$ for helium accretors (Strohmayer \& Brown 2002). These authors find that, for $\dot{M} \lesssim 0.3 \dot{M}_{\text {Edd }}$, a smaller mass fraction of carbon than these limits will not produce a superburst. Especially for mixed hydrogen/helium accretors, the primary cause of the uncertainty in the carbon fraction is due to the rp-process (Wallace \& Woosley 1981). Several research groups have studied the final products of the rp-process from both stable and unstable burning (Schatz et al. 1999, 2003b; Koike et al. 2004; Woosley et al. 2004; Fisker et al. 2004). They find that the carbon mass fraction of the ashes is notably below $10 \%$ for the range of accretion rates at which superbursts have been observed, which is problematic since such a low fraction is insufficient to trigger a superburst. The exception to this statement may be the system GX 17+2, in which extremely energetic Type I X-ray bursts were observed at accretion rates in the neighborhood of the Eddington limit (Kuulkers et al. 2002a; in't Zand et al. 2004a). Despite these results, we cannot rule out the possibility that some other process (e.g., delayed mixed bursts, see §5) may still be able to produce enough carbon. Due to this uncertainty, we take as a free parameter $C_{\mathrm{f}}$, the fraction of hydrogen and helium that ultimately burns to carbon. 
Figure 9 shows superburst energies and recurrence times as a function of accretion rate for three choices of $C_{\mathrm{f}}$. For a given accretion rate, the superburst recurrence time will usually be shorter for a larger mass fraction of carbon at the base of the accreted layer because an instability will occur sooner if more carbon is present. To first order, the burst energy $E_{\text {burst }} \propto \Sigma_{\text {layer }} C_{\mathrm{f}}$, where $\Sigma_{\text {layer }}$ is the column depth of the accreted layer when a superburst is triggered. However, $\Sigma_{\text {layer }}$ itself is roughly proportional to the recurrence time, so it is difficult to derive a general relationship between $C_{\mathrm{f}}$ and $E_{\text {burst }}$ (compare the burst energies for $C_{\mathrm{f}}=0.3$ and $C_{\mathrm{f}}=0.5$ in Figure 9 ). In general, the value of $C_{\mathrm{f}}$ does not substantially affect superburst energetics and recurrence times for a given accretion rate. However, the value of $C_{\mathrm{f}}$ does significantly affect the lower limit of the range of accretion rates at which superbursts occur. We find that the lower limit is roughly inversely proportional to $C_{\mathrm{f}}$, in agreement with Cumming \& Bildsten (2001).

As noted in $§ 3.3$, the composition of the heavy elements in the accreted layer has a negligible affect on the thermal profile of the superburst ignition region. Consequently, for accretion rates at which superbursts are triggered, superburst energies and recurrence times are quite insensitive to the nuclear composition of the heavy elements in the accreted layer. This agrees well with the results of Brown (2004). At lower accretion rates, however, the greater charge of the ${ }_{44}^{104} \mathrm{Ru}$ ions may cause the base of the accreted layer to solidify before a thermonuclear instability can occur. In this case, the carbon fuel burns stably via pycnonuclear reactions (see $§ 2.3 .2$ ), so a superburst does not occur. Therefore, though the composition of the heavy elements in the ocean is unimportant with regard to superburst characteristics, it is important with regard to the presence or absence of superbursts at a given accretion rate. This dependence has not been noted in previous studies. See Figure 10

for a plot of superburst energies and recurrence times for neutron star crusts with different heavy element compositions.

\subsection{Neutrino Cooling Mechanism in the Core}

The composition of dense matter in the inner cores of neutron stars is essentially unknown. However, this composition significantly affects the neutrino emission there. Thus, knowledge of which neutrino processes occur in the core can help constrain the types of matter that exist at such high densities.

When we solve for the core temperature by balancing the flux flowing inward with the neutrino cooling, we find $T_{\text {core }} \approx 3 \times 10^{8} \mathrm{~K}$ for nonsuperfluid cores that emit neutrinos via modified URCA reactions and $T_{\text {core }} \approx 2 \times 10^{7} \mathrm{~K}$ for cores that emit neutrinos via pionic reactions. In general, $T_{\text {core }} \propto \dot{M}^{1 / 8}$ for modified URCA cooling and $T_{\text {core }} \propto \dot{M}^{1 / 6}$ for pionic 
cooling. The thermal profile of much of the crust, and in particular the region in which superbursts are triggered, is quite sensitive to the core temperature (see Fig. 5). Thus, compared to stars with inefficient cooling mechanisms in their cores, neutron stars with efficient cooling mechanisms in their cores need to accrete more fuel in order for the carbon at the base of the accreted layer to ignite. Consequently, these superbursts will be more energetic and will have longer recurrence times, as shown in Figure 11.

If neutrons and protons exist in the stellar core, they probably become superfluid at high densities (Baym et al. 1969). Baryon superfluidity drastically suppresses neutrino emission from modified and direct URCA processes and therefore raises the temperature in the core (Yakovlev et al. 1999, 2001; Yakovlev \& Pethick 2004). Due to the large uncertainties in the composition and physical state of the matter, we do not attempt to model the superfluid core in detail. Rather, to study the effects of superfluidity on superburst characteristics, we simply fix the temperature at the crust-core interface at several values and carry out the calculations. Figure 12 shows the results. Neutron stars with hotter cores exhibit superbursts with smaller fluences and shorter recurrence times. In addition, we see that for these temperatures the lower limit of the accretion rate range over which superbursts occur increases as the core temperature increases. The high crust temperature causes the carbon to burn stably before an instability is triggered, in agreement with Cumming \& Bildsten (2001).

Impurities in the crust of a neutron star can significantly affect the thermal profile of the superburst ignition region, especially in stars with cold cores (see §3.5). Consequently, superburst energies and recurrence times are quite sensitive to the concentration of impurities in the crust. Figures 13 and 14 show some results. We see that there is little difference between $Q=0$ and $Q=5.2$ when the core radiates neutrinos via modified URCA reactions, but $Q=100$ induces a substantial change in the results. However, recent investigations suggest that impurity scattering in the crusts of accreting neutron stars that exhibit superbursts is rather insignificant (Schatz et al. 2003a). If the impurity concentration $Q \sim 1$ in the crust of a neutron star with a core that emits neutrinos via pionic or direct URCA reactions, then the carbon fuel will solidify and burn stably via pycnonuclear reactions at lower accretion rates.

We have shown in $§ 3.6$ that, for a completely disordered neutron star crust, the thermal profile (including that of the superburst ignition region) is highly insensitive to the core neutrino emission mechanism. In this case, superburst energies and recurrence times should be insensitive to the core cooling mechanism, as confirmed in Figure 15. Thus we verify the result of Brown (2004) that superburst energies and recurrence times from neutron stars with highly efficient core neutrino cooling mechanisms may still be consistent with observations 
if the crust is disordered. However, in this case we find that superbursts should occur even at relatively low accretion rates $\dot{M}<0.1 \dot{M}_{\text {Edd }}$, whereas observations indicate a cutoff

at $\dot{M} \approx 0.1 \dot{M}_{\text {Edd }}$. Normally, the carbon would burn stably at these low accretion rates because the energy generated would be efficiently transported away from the burning region. However, the low thermal conductivity due to the disordered lattice inhibits the flow of energy away from the carbon-burning region. Therefore, even a low carbon energy generation rate can initiate a thermonuclear instability. Note that in deriving these results we assume that the entire crust is completely disordered, which is clearly an extreme situation. Further investigations into the nuclear structure of neutron star crusts are necessary to determine the significance of these results.

\subsection{Stellar Radius}

The radius of a neutron star depends quite sensitively on the core equation of state, but it is virtually independent of the stellar mass. Accurate measurements of the radius to within about one kilometer can potentially constrain the equation of state (Lattimer \& Prakash 2001). To demonstrate the effects of the stellar radius on superburst characteristics, we choose three different values, $R=16.4,10.4$, and $6.5 \mathrm{~km}$, which likely bracket the true radii of neutron stars. We find that stars with larger radii have more energetic superbursts and longer recurrence times at a given accretion rate. They also have superbursts at lower accretion rates. See Figure 16.

At a given accretion rate, a neutron star with a larger radius requires a larger column density of fuel in order for a superburst to be triggered. This is a result of the lower gravitational acceleration near the stellar surface. Thus the effect of radius on superburst recurrence times is twofold. Not only is the accretion rate per unit area smaller for a larger star, but also the amount of fuel per unit area required for a superburst to occur is larger. The effect of radius on superburst energetics is even stronger. In addition to the factors stated above, the total surface area of the star is larger, so the total amount of fuel available to burn at a given column depth is greater. Figure 16 thus suggests that superburst observations may be useful for constraining neutron star radii.

\section{Comparison with Observations}

As noted in $\S 1$, the nine superbursts that have been observed, excluding those from GX $17+2$, have integrated photon fluxes of $\approx 10^{42}$ ergs. The paucity of data makes the 
recurrence time of superbursts difficult to determine, but observations imply a recurrence time of $\sim 1-2$ years. All nine superbursts occurred in systems with accretion rates between $10 \%$ and $30 \%$ of the Eddington limit. Additionally, several superburst candidates have been observed in the near-Eddington accretor GX 17+2 with energies of $\sim 5 \times 10^{41}$ ergs. A successful theoretical model of superbursts must explain these facts.

We begin by discussing the $\dot{M}$ range over which superbursts are observed. The lack of superbursts for accretion rates $\dot{M} \lesssim 0.1 \dot{M}_{\text {Edd }}$ arises naturally in our model and is rather simple to explain theoretically. As discussed in $\S 4.3$, at low $\dot{M}$, either the carbon fuel burns stably via thermonuclear reactions before an instability is triggered (for high core temperatures, Cumming \& Bildsten 2001) or the crust solidifies in the superburst ignition region and the carbon burns stably via pycnonuclear reactions (for low core temperatures). In either case, there is a cutoff of superbursts at low values of $\dot{M}$.

The above discussion is invalid if the crust is highly disordered, since then the thermal conductivity is low, causing the region in which superbursts are triggered to be hotter than if the crust were ordered. Also, when the carbon ignites, the low thermal conductivity inhibits the diffusion of the nuclear energy generated there. The nuclear energy generation rate exceeds the rate at which thermal conduction can cool the region, so a thermonuclear runaway ensues and a superburst is triggered. Therefore, as seen in Figure 15, superbursts occur down to accretion rates below $0.1 \dot{M}_{\text {Edd }}$. The lack of observed superbursts for accretion rates $\dot{M} \lesssim 0.1 \dot{M}_{\text {Edd }}$ then needs to be explained. One possibility is that, for some reason, e.g., lack of delayed mixed bursts (see below), carbon is not produced in sufficient quantities to fuel a superburst. Another explanation could be that at low accretion rates the recurrence times are so long that astronomers simply have not observed any of the systems long enough to see superbursts.

The absence of superbursts for $0.3 \dot{M}_{\text {Edd }} \lesssim \dot{M} \lesssim 1.0 \dot{M}_{\text {Edd }}$ is not well understood. Every theoretical study, including our own, suggests that superbursts should occur more easily at higher mass accretion rates, assuming a sufficient amount of carbon is present. The peak luminosities of superbursts are often below the Eddington limit. Therefore, one possible explanation is that none has been observed in this range of accretion rates because there is little contrast between the peak burst luminosity and the accretion luminosity (Strohmayer $\&$ Bildsten 2004). On the other hand, the recurrence time is inversely proportional to the accretion rate to first order, so one would expect to observe more superbursts at higher accretion rates.

Another possible explanation involves the rp-process. Perhaps the rp-process leaves behind enough unburnt carbon to produce superbursts only for $\dot{M}<0.3 \dot{M}_{\text {Edd }}$, and it burns too much carbon at higher mass accretion rates. Both the critical amount of carbon needed 
for superbursts and the amount of unburnt carbon left by the rp-process decrease with increasing $\dot{M}$. Perhaps the two dependencies conspire to permit superbursts only for $\dot{M}<$ $0.3 \dot{M}_{\mathrm{Edd}}$. One problem with this explanation is that all studies of the rp-process carried out so far predict too little carbon to produce superbursts at all $\dot{M}$.

The above proposals do not explain the observed superbursts from GX 17+2, which accretes at $\dot{M} \sim \dot{M}_{\text {Edd }}$. If one can observe superbursts in a system accreting at nearly the Eddington limit, than presumably one could observe superbursts in systems accreting at any lower rate as well. Furthermore, as discussed in $\S 4.2$, the minimum mass fraction of carbon needed to trigger a superburst is inversely proportional to the accretion rate. However, the amount of carbon left behind by stable burning via the rp-process falls even more rapidly with increasing $\dot{M}$.

Yet another possible explanation involves ideas described in Paper I. In that paper, Narayan and Heyl showed that at high accretion rates $\left(\dot{M} \gtrsim 0.1 \dot{M}_{\text {Edd }}\right)$ normal Type I Xray bursts occur in a unique regime that they refer to as "delayed mixed bursts." In these systems, a large fraction of the hydrogen and helium fuel burns stably to carbon before the instability is triggered. This stable burning explains the observations of van Paradijs et al. (1988) who found that, for systems that accrete at a high rate and exhibit normal bursts, the quantity $\alpha$, the ratio of the energy released between bursts to the energy released during a burst, rises dramatically. Such an increase of $\alpha$ is nicely reproduced by the model described in Paper I. Furthermore, in’t Zand et al. (2003) showed that systems that exhibit superbursts generally have $\alpha$ values that are significantly greater than systems that do not exhibit superbursts.

Delayed mixed bursts may be the source of the substantial amount of carbon needed to trigger a superburst. As noted earlier, every system in which a superburst has been observed exhibits normal bursts as well. We tentatively suggest that the occurrence of delayed mixed bursts is a necessary condition for superbursts in systems for which the accreted material is predominantly hydrogen. The stably burned material forms a thick layer of carbon, and the delayed burst that finally occurs burns above this layer, leaving the carbon largely unaffected. This would explain the absence of observed superbursts in systems with accretion rates $0.3 \dot{M}_{\mathrm{Edd}} \lesssim \dot{M} \lesssim 1.0 \dot{M}_{\mathrm{Edd}}$. These systems do not exhibit normal Type I bursts, so the hydrogen and helium fuel burns stably via the rp-process. According to estimates in the literature, the rp-process leaves behind too little carbon for a superburst. Note that the existence of normal bursts in GX $17+2$ is not understood theoretically if the accreted material is indeed predominantly hydrogen. However, Kuulkers et al. (2002b) find that $\alpha \gtrsim 1000$ in this system, which implies that the normal bursts are delayed bursts. We emphasize that this explanation is merely a hypothesis, and it is not without issues. In 
particular, we do not know if the carbon produced during the stable burning phase of a delayed burst system would survive when the burst is triggered above it. More research is needed on this interesting problem.

Delayed mixed bursts may also explain the absence of superbursts for $\dot{M}<0.1 \dot{M}_{\text {Edd }}$ in models with disordered crusts. These models predict superbursts down to relatively low accretion rates, provided they have enough carbon. However, Paper I showed that at accretion rates below $0.1 \dot{M}_{\text {Edd }}$ the normal Type I bursts are prompt bursts. Such bursts are expected to burn all the fuel to elements much heavier than carbon, and so their ashes should not support superbursts.

We next consider the energies and recurrence times of superbursts. Leaving aside models with disordered crusts, Figure 11 shows that neutron stars with ordered crusts and highly efficient neutrino emission mechanisms in their cores have much more powerful superbursts and longer recurrence times than are observed. Furthermore, if the impurity parameter $Q \sim 1$, these stars do not exhibit superbursts at accretion rates $\dot{M} \lesssim 0.3 \dot{M}_{\text {Edd }}$, where all superbursts except those from GX $17+2$ have been observed (see Fig. 14). Therefore, we conclude that accreting neutron stars with highly efficient neutrino emission in their cores (due to direct URCA or pionic reactions, for example) are inconsistent with superburst observations, in agreement with Brown (2004).

Even for neutron stars that emit neutrinos via modified URCA reactions in their cores, we predict energies and recurrence times somewhat larger than those observed. The observed superburst energies are $\sim 10^{42} \mathrm{ergs}$, which is roughly an order of magnitude lower than those in Figure 11 for modified URCA cooling. However, a substantial fraction of the superburst energy may be released in the form of neutrinos (Strohmayer \& Brown 2002), so this discrepancy may not be serious. Potentially more troublesome are the relatively long superburst recurrence times predicted by the model. Although observations do not constrain recurrence times very well, the times are probably shorter than those in Figure 11. Neutron star models with hot cores, $T_{\text {core }} \gtrsim 5 \times 10^{8} \mathrm{~K}$, seem to best match the observational data, though the $\dot{M}$ cutoff is larger (see Figure 12). Such temperatures correspond to neutrino cooling that is even less efficient than that from modified URCA reactions. One possibility is that a nonnegligible fraction of the neutron star core consists of superfluid baryonic matter.

The above conclusions about the neutrino emission mechanism in the core assume that the stellar radius is the canonical $10 \mathrm{~km}$. The radius of the neutron star is the only other parameter which we have investigated that significantly affects superburst characteristics at the accretion rates at which superbursts are observed. Larger stars produce superbursts with larger energetics and longer recurrence times (see Figure 16). Neutron stars with exceptionally large radii $(R \approx 16.4 \mathrm{~km})$ produce extremely energetic superbursts that are 
grossly inconsistent with observations, even if the core temperature is very high. We cannot make such a definitive statement about neutron stars with smaller radii because we are unable to differentiate between superbursts from neutron stars with small radii $(R \approx 6.5$ $\mathrm{km})$ and moderate core temperatures $\left(T_{\text {core }} \approx 3 \times 10^{8} \mathrm{~K}\right)$ and neutron stars with moderate radii $(R \approx 10.4 \mathrm{~km})$ and high core temperatures $\left(T_{\text {core }} \approx 8 \times 10^{8} \mathrm{~K}\right)$.

\section{Comparison with Previous Theoretical Work}

Previous theoretical investigations of the superburst phenomenon (Brown \& Bildsten 1998, Cumming \& Bildsten 2001, Strohmayer \& Brown 2002, Brown 2004) generally agree quite well with our results. To determine the physical conditions under which superbursts occur, the authors use approximate ignition criteria evaluated at the base of the accreted layer. In this section, we compare and contrast the results of our rigorous global linear stability analysis with those obtained from these approximate one-zone ignition criteria.

According to Brown \& Bildsten (1998), an instability ensues when the carbon nuclear energy generation rate $\epsilon_{\mathrm{C}}$ at the base of the accreted layer satisfies the criterion

$$
\frac{\mathrm{d} \epsilon_{\mathrm{C}}}{\mathrm{d} T}>\frac{\mathrm{d} \epsilon_{\mathrm{cool}}}{\mathrm{d} T}
$$

where $\epsilon_{\text {cool }}=\rho K T / \Sigma^{2}$ is an approximation to the global cooling rate and $K$ is the thermal conductivity evaluated at the base of the accreted layer. Cumming \& Bildsten (2001) and Brown (2004) set $\mathrm{d} \ln \epsilon_{\mathrm{C}} / \mathrm{d} \ln T=26$ and $\mathrm{d} \ln \epsilon_{\mathrm{cool}} / \mathrm{d} \ln T=2$ and express the ignition criterion as $\epsilon_{\mathrm{C}}>(2 / 26) \epsilon_{\mathrm{cool}}$. We presume that Brown \& Bildsten (1998) and Strohmayer \& Brown (2002) calculate the derivatives numerically. Unlike Brown \& Bildsten (1998) and Strohmayer \& Brown (2002), Cumming \& Bildsten (2001) and Brown (2004) do not explicitly state that they track the evolution of the carbon mass fraction $Z_{\mathrm{CNO}}$ when they solve for the equilibrium configuration of the layer. To our knowledge, these authors keep $Z_{\mathrm{CNO}}$ constant throughout the layer, but we cannot state this with certainty.

To conduct an accurate comparison between our global analysis and the various onezone approximations, we solve for the equilibrium configuration of the accreted layer in an identical fashion for both methods. This ensures that any differences in the results are due only to the stability calculation. We find that $\mathrm{d} \ln \epsilon_{\mathrm{cool}} / \mathrm{d} \ln T \approx 2$ in all of our calculations. Therefore, setting $\mathrm{d} \ln \epsilon_{\mathrm{cool}} / \mathrm{d} \ln T=2$ is appropriate for all scenarios in our opinion. In contrast, we find that setting $\mathrm{d} \ln \epsilon_{\mathrm{C}} / \mathrm{d} \ln T=26$ is appropriate only if the core temperature is high $\left(\gtrsim 10^{8} \mathrm{~K}\right)$ or the accretion rate is near the Eddington limit. At sufficiently high densities and low temperatures, $\mathrm{d} \ln \epsilon_{\mathrm{C}} / \mathrm{d} \ln T \ll 26$, often by many orders of magnitude, so this approximation is inappropriate in these situations. 
For a given equilibrium configuration, we calculate the one-zone approximation in four different ways. When calculating $\mathrm{d} \epsilon_{\mathrm{C}} / \mathrm{d} T$ at the base of the accreted layer we (i) use the value of $Z_{\mathrm{CNO}}$ and $\mathrm{d} \ln \epsilon_{\mathrm{C}} / \mathrm{d} \ln T$ derived from our equilibrium configuration, (ii) artificially set $Z_{\mathrm{CNO}}$ constant and use the value of $\mathrm{d} \ln \epsilon_{\mathrm{C}} / \mathrm{d} \ln T$ derived from our equilibrium configuration, (iii) use the value of $Z_{\mathrm{CNO}}$ derived from our equilibrium configuration and set $\mathrm{d} \ln \epsilon_{\mathrm{C}} / \mathrm{d} \ln T=$ 26 , or (iv) artificially set $Z_{\mathrm{CNO}}$ constant and set $\mathrm{d} \ln \epsilon_{\mathrm{C}} / \mathrm{d} \ln T=26$. Artificially setting $Z_{\mathrm{CNO}}$ constant results in superbursts at all accretion rates for each calculation we performed. This is clearly incorrect since all our global stability analysis models indicate a minimum $\dot{M}$ below which superbursts are absent. Therefore, the criteria (ii) and (iv) above are very inaccurate and should be avoided. Regarding the other two criteria, we find that for neutron stars with high core temperatures, such as those with cores that radiate neutrinos via modified URCA reactions, the results from the calculations in which $\mathrm{d} \ln \epsilon_{\mathrm{C}} / \mathrm{d} \ln T$ is set to 26 and in which $\mathrm{d} \ln \epsilon_{\mathrm{C}} / \mathrm{d} \ln T$ is calculated self-consistently are almost identical. Therefore, the approximation $\mathrm{d} \ln \epsilon_{\mathrm{C}} / \mathrm{d} \ln T=26$ is valid in this situation. However, for neutron stars with low core temperatures, such as those with cores that radiate neutrinos via pionic reactions, the results from the two calculations differ significantly, so in this case $\mathrm{d} \ln \epsilon_{\mathrm{C}} / \mathrm{d} \ln T$ must be calculated self-consistently.

See Figures 17 and 18 for a comparison between the results from our global linear stability analysis and the one-zone approximations (i) and (iii). For the calculations in which both $Z_{\mathrm{CNO}}$ and $\mathrm{d} \ln \epsilon_{\mathrm{C}} / \mathrm{d} \ln T$ are calculated self-consistently, the results of the one-zone approximation and the global linear stability analysis generally agree quite well. The errors produced by the one-zone analysis are probably smaller than those due to uncertainties in other parameters, such as the accretion rate, impurity concentration, elemental composition of the crust, etc. The only significant discrepancy we find between the two models is the superburst recurrence times in systems with accretion rates near the critical rate below which superbursts do not occur. In these systems, we find from our global linear stability analysis that the carbon layer is marginally unstable. A large fraction of the carbon fuel burns stably before the full instability is triggered, resulting in a delayed burst (see Paper I), whereas the one-zone calculation has no way of modeling delayed bursts. These "delayed" superbursts have significantly larger recurrence times than those derived using the one-zone approximation. However, they occur over a fairly narrow range of accretion rates, so this discrepancy may not be serious. From our comparison, we draw the following conclusions regarding the application of the one-zone approximation to superburst calculations: The one-zone approximation is appropriate for most superburst calculations if one uses the values of $Z_{\mathrm{CNO}}$ and $\mathrm{d} \ln \epsilon_{\mathrm{C}} / \mathrm{d} \ln T$ derived from the equilibrium configuration calculation. The resulting superburst energies should be sufficiently accurate for all accretion rates. The superburst recurrence times should be sufficiently accurate at high accretion rates, but they will 
significantly underestimate the true recurrence times at accretion rates near the critical rate. All simpler approximations, such as (ii), (iii), and (iv) above, make serious errors for some choices of parameters.

\section{Summary}

In this investigation, we have carried out the first self-consistent global linear stability analysis of the carbon fuel on accreting neutron stars and determined the physical conditions under which superbursts occur. Our model reproduces the general observed superburst features, including burst energies, recurrence times, and the range of accretion rates at which superbursts occur. In contrast to normal Type I X-ray bursts, the observational characteristics of superbursts are very sensitive to the thermal profile of the entire crust of the neutron star. Consequently, superbursts can be useful probes to study neutron star interiors. Because our theoretical model evaluates the thermal profile self-consistently, we are able to explore a wide range of neutron star parameters and study the effects of each on the resulting superburst energies and recurrence times.

By comparing our results with observations, we are able to set constraints on various neutron star parameters. We find that accreting neutron stars with highly efficient neutrino emission in their cores produce extremely energetic superbursts which are inconsistent with observations. Such neutron stars also do not have superbursts in the range of accretion rates at which superbursts are observed unless the crust is very impure. Stars with less efficient neutrino emission produce bursts that agree better with observations, while stars with highly suppressed neutrino emission in their cores, e.g., because of superfluidity, produce bursts that agree best with observations.

If the neutron star crust is disordered, the thermal profile of the crust, in particular the region in which superbursts are triggered, is insensitive to the core temperature. Therefore, superburst energetics and recurrence times from neutron stars with highly efficient core neutrino cooling mechanisms may still be consistent with observations if the crust is completely disordered. However, such neutron stars will have superbursts at accretion rates lower than the observed cutoff rate. The cutoff at $\dot{M} \sim 0.1 \dot{M}_{\text {Edd }}$ thus requires some other effect, e.g. delayed mixed bursts.

Neutron stars with large radii $(R \sim 16 \mathrm{~km})$ produce very energetic superbursts that are inconsistent with observations, even if the core neutrino emission mechanism is highly inefficient. Better constraints on neutron star parameters could be made with improvements to the theoretical model and with more observational data. 
All systems in which superbursts are observed and that accrete predominantly hydrogen have exceptionally high $\alpha$ values (see $\S 5$ ). Delayed mixed bursts, which are normal bursts that burn a significant amount of hydrogen and helium stably to carbon before the instability is triggered (Paper I), occur in systems for which $0.1 \lesssim \dot{M} / \dot{M}_{\text {Edd }} \lesssim 0.3$ and produce $\alpha$ values consistent with those observed in these systems. Since the delayed mixed burst instability occurs above a layer of carbon that has been already produced by stable burning, it is possible that they leave behind sufficient amounts of unburnt carbon to fuel superbursts. For systems in which delayed mixed bursts do not occur, it is likely that the rp-process burns nearly all the available fuel to elements heavier than carbon, leaving insufficient carbon for superbursts. We thus speculate that delayed mixed bursts are a necessary prerequisite for the occurrence of superbursts in systems that accrete predominantly hydrogen. More work is needed to verify this suggestion.

Previous theoretical investigations have used approximate one-zone ignition criteria to determine the conditions under which superburst occur. We find that the results of these approximate criteria generally agree well with our global stability analysis if the parameters used in the one-zone criteria are consistent with those derived in the equilibrium configuration calculation. Specifically, calculations in which the carbon mass fraction is kept constant do not reproduce the absence of superbursts at lower accretion rates, and calculations which set $\mathrm{d} \ln \epsilon_{\mathrm{C}} / \mathrm{d} \ln T=26$ produce inaccurate results for neutron stars with highly efficient neutrino emission mechanisms in their cores. For calculations in which these quantities are consistent with those derived in the equilibrium configuration calculation, the only significant discrepancy we have found is for systems that accrete at a rate close to the critical accretion rate below which superbursts do not occur. At these accretion rates, a substantial amount of carbon burns stably before a superburst is triggered. Consequently, the one-zone approximation drastically underestimates the superburst recurrence times.

It is our pleasure to thank Edward Brown, Jean in't Zand, and Don Lamb for helpful

discussions and the referee for insightful comments and suggestions. This work was supported by NASA grant NNG04GL38G.

\section{REFERENCES}

Ashcroft, N. \& Mermin, N. 1976, Solid State Physics (Philadelphia: Saunders College)

Baiko, D. A., \& Yakovlev, D. G. 1995, Astronomy Letters, 21, 702

Baym, G., Pethick, C., \& Pines, D. 1969, Nature, 224, 673 
Brown, E. F. 2000, ApJ, 531, 988

—. 2004, ApJ, 614, L57

Brown, E. F. \& Bildsten, L. 1998, ApJ, 496, 915

Brown, E. F., Bildsten, L., \& Rutledge, R. E. 1998, ApJ, 504, L95

Caughlan, G. R. \& Fowler, W. A. 1988, Atomic Data and Nuclear Data Tables, 40, 283

Chabrier, G. 1993, ApJ, 414, 695

Chabrier, G., \& Potekhin, A. Y. 1998, Phys. Rev. E, 58, 4941

Clayton, D. D. 1983, Principles of stellar evolution and nucleosynthesis (Chicago: University of Chicago Press, 1983)

Cooper, R. L. \& Narayan, R. 2004, AAS/High Energy Astrophysics Division, 8

Cornelisse, R., Heise, J., Kuulkers, E., Verbunt, F., \& in’t Zand, J. J. M. 2000, A\&A, 357, L21

Cumming, A. 2003, ApJ, 595, 1077

Cumming, A. \& Bildsten, L. 2001, ApJ, 559, L127

Farouki, R. T. \& Hamaguchi, S. 1993, Phys. Rev. E, 47, 4330

Fedorova, A. V. \& Ergma, E. V. 1989, Ap\&SS, 151, 125

Fisker, J. L., Brown, E., Liebendoerfer, M., Schatz, H., \& Thielemann, F. -. 2004, ArXiv Astrophysics e-prints, astro-ph/0407319

Friman, B. L. \& Maxwell, O. V. 1979, ApJ, 232, 541

Fushiki, I. \& Lamb, D. Q. 1987, ApJ, 323, L55

Haensel, P., Kaminker, A. D., \& Yakovlev, D. G. 1996, A\&A, 314, 328

Haensel, P. \& Zdunik, J. L. 1990a, A\&A, 229, 117

-. 1990b, A\&A, 227, 431

-. 2003, A\&A, 404, L33

in't Zand, J. J. M., Cornelisse, R., \& Cumming, A. 2004a, A\&A, 426, 257 
in't Zand, J. J. M., Cornelisse, R., Kuulkers, E., Heise, J., Verbunt, F., \& Cumming, A. 2004b, AAS/High Energy Astrophysics Division, 8

in't Zand, J. J. M., Kuulkers, E., Verbunt, F., Heise, J., \& Cornelisse, R. 2003, A\&A, 411, L487

Itoh, N., Hayashi, H., Nishikawa, A., \& Kohyama, Y. 1996, ApJS, 102, 411

Itoh, N. \& Kohyama, Y. 1993, ApJ, 404, 268

Kitamura, H. 2000, ApJ, 539, 888

Koike, O., Hashimoto, M., Kuromizu, R., \& Fujimoto, S. 2004, ApJ, 603, 242

Kuulkers, E. 2004, Nuclear Physics B Proceedings Supplements, 132, 466

Kuulkers, E., Homan, J., van der Klis, M., Lewin, W. H. G., \& Méndez, M. 2002a, A\&A, 382,947

Kuulkers, E., in't Zand, J. J. M., van Kerkwijk, M. H., Cornelisse, R., Smith, D. A., Heise, J., Bazzano, A., Cocchi, M., Natalucci, L., \& Ubertini, P. 2002b, A\&A, 382, 503

Kuulkers, E., in’t Zand, J., Homan, J., van Straaten, S., Altamirano, D., \& van der Klis, M. 2004, AIP Conf. Proc. 714: X-ray Timing 2003: Rossi and Beyond, 714, 257

Landau, L. D. \& Lifshitz, E. M. 1969, Statistical physics. Pt.1 (Course of theoretical physics Pergamon International Library of Science, Technology, Engineering and Social Studies, Oxford: Pergamon Press, and Reading: Addison-Wesley, -c1969, 2nd rev. enlarg.ed.)

Lattimer, J. M. \& Prakash, M. 2001, ApJ, 550, 426

Mackie, F. D. \& Baym, G. 1977, Nuclear Physics A, 285, 332

Magierski, P. \& Bulgac, A. 2004, Nuclear Physics A, 738, 143

Magierski, P. \& Heenen, P.-H. 2002, Phys. Rev. C, 65, 045804

Maxwell, O., Brown, G. E., Campbell, D. K., Dashen, R. F., \& Manassah, J. T. 1977, ApJ, 216,77

Misner, C. W., Thorne, K. S., \& Wheeler, J. A. 1973, Gravitation (San Francisco: W.H. Freeman and Co., 1973)

Narayan, R. \& Heyl, J. S. 2003, ApJ, 599, 419 (Paper I) 
Negele, J. W. \& Vautherin, D. 1973, Nuclear Physics A, 207, 298

Ogata, S., Ichimaru, S., \& van Horn, H. M. 1993, ApJ, 417, 265

Podsiadlowski, P., Rappaport, S., \& Pfahl, E. D. 2002, ApJ, 565, 1107

Potekhin, A. Y., Baiko, D. A., Haensel, P., \& Yakovlev, D. G. 1999, A\&A, 346, 345

Schatz, H., Bildsten, L., Cumming, A., \& Wiescher, M. 1999, ApJ, 524, 1014

Schatz, H., Aprahamian, A., Barnard, V., Bildsten, L., Cumming, A., Ouellette, M., Rauscher, T., Thielemann, F.-K., \& Wiescher, M. 2001, Physical Review Letters, 86, 3471

Schatz, H., Bildsten, L., \& Cumming, A. 2003a, ApJ, 583, L87

Schatz, H., Bildsten, L., Cumming, A., \& Ouellette, M. 2003b, Nuclear Physics A, 718, 247

Schatz, H., Bildsten, L., Cumming, A., \& Wiescher, M. 1999, ApJ, 524, 1014

Shapiro, S. L. \& Teukolsky, S. A. 1983, Black holes, white dwarfs, and neutron stars: The physics of compact objects (Research supported by the National Science Foundation. New York, Wiley-Interscience, 1983, 663 p.)

Strohmayer, T. \& Bildsten, L. 2004, in Compact Stellar X-Ray Sources, ed. W. H. G. Lewin and M. van der Klis (Cambridge: Cambridge Univ. Press), in press (astro$\mathrm{ph} / 0301544)$

Strohmayer, T. E. 2000, Bulletin of the American Astronomical Society, 32, 1218

Strohmayer, T. E. \& Brown, E. F. 2002, ApJ, 566, 1045

Strohmayer, T. E. \& Markwardt, C. B. 2002, ApJ, 577, 337

Taam, R. E. \& Picklum, R. E. 1978, ApJ, 224, 210

Thorne, K. S. 1977, ApJ, 212, 825

van Paradijs, J., Penninx, W., \& Lewin, W. H. G. 1988, MNRAS, 233, 437

Wallace, R. K. \& Woosley, S. E. 1981, ApJS, 45, 389

Wijnands, R. 2001, ApJ, 554, L59

Woosley, S. E., Heger, A., Cumming, A., Hoffman, R. D., Pruet, J., Rauscher, T., Fisker, J. L., Schatz, H., Brown, B. A., \& Wiescher, M. 2004, ApJS, 151, 75 
Woosley, S. E. \& Taam, R. E. 1976, Nature, 263, 101

Yakovlev, D. G. \& Kaminker, A. D. 1996, Astronomy Letters, 22, 491

Yakovlev, D. G., Kaminker, A. D., Gnedin, O. Y., \& Haensel, P. 2001, Phys. Rep., 354, 1

Yakovlev, D. G. \& Levenfish, K. P. 1995, A\&A, 297, 717

Yakovlev, D. G., Levenfish, K. P., \& Shibanov, Y. A. 1999, Uspekhi Fizicheskikh Nauk, 42, 737

Yakovlev, D. G. \& Pethick, C. J. 2004, ARA\&A, 42, 169 


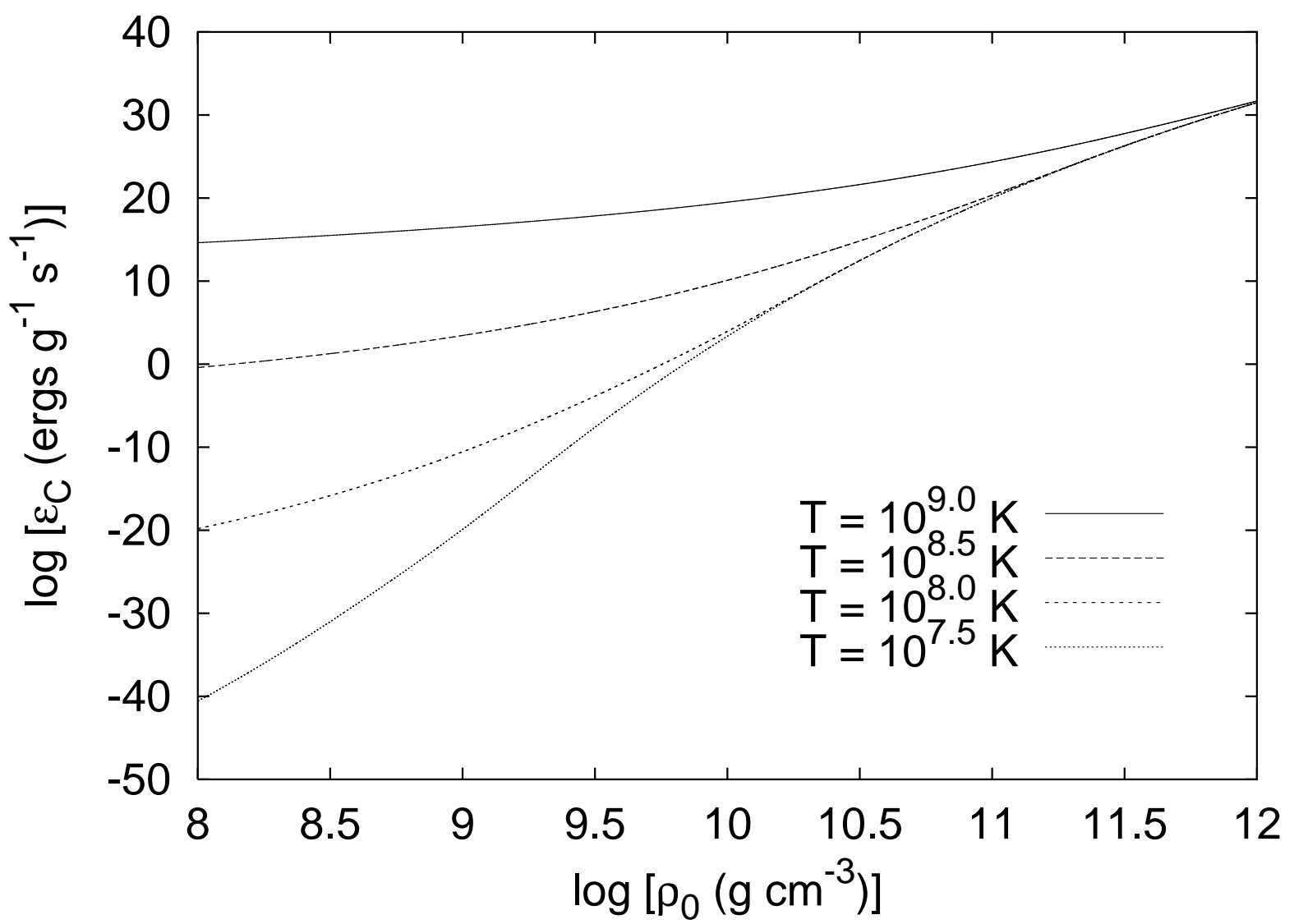

Fig. 1.- Carbon energy generation rates as a function of rest mass density, plotted for four different temperatures. The composition assumed is $30 \%{ }^{12} \mathrm{C}$ and $70 \%{ }^{56} \mathrm{Fe}$ by mass. Note that at high densities, when the burning is strictly pycnonuclear, the energy generation rate is essentially independent of temperature. 

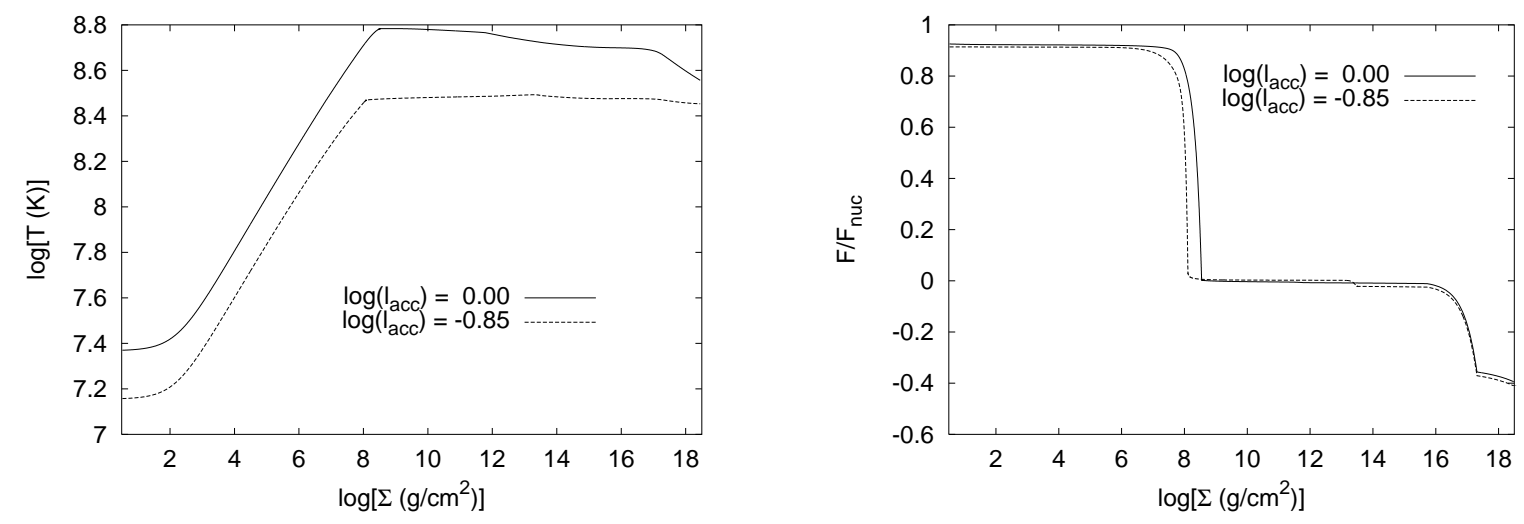

Fig. 2.- Thermal and net energy flux profiles for two neutron stars with different mass accretion rates just before a superburst is triggered. The parameter $l_{\text {acc }}$ is the accretion rate normalized to the Eddington limit. The rate of accretion of matter onto the surface of a neutron star significantly affects the thermal state of the entire crust, as well as the stellar core. For this plot we assume fiducial parameter values $M=1.4 M_{\odot}, R=10.4 \mathrm{~km}$, $C_{\mathrm{f}}=0.3, X_{\text {out }}=0.7, Y_{\text {out }}=0.28$, and $Z_{\mathrm{CNO}, \text { out }}=0.016$. Additionally, we assume that the heavy element in the accreted layer is ${ }^{56} \mathrm{Fe}$ and that the core emits neutrinos via modified URCA reactions. These assumptions hold for all other Figures unless notified otherwise. 


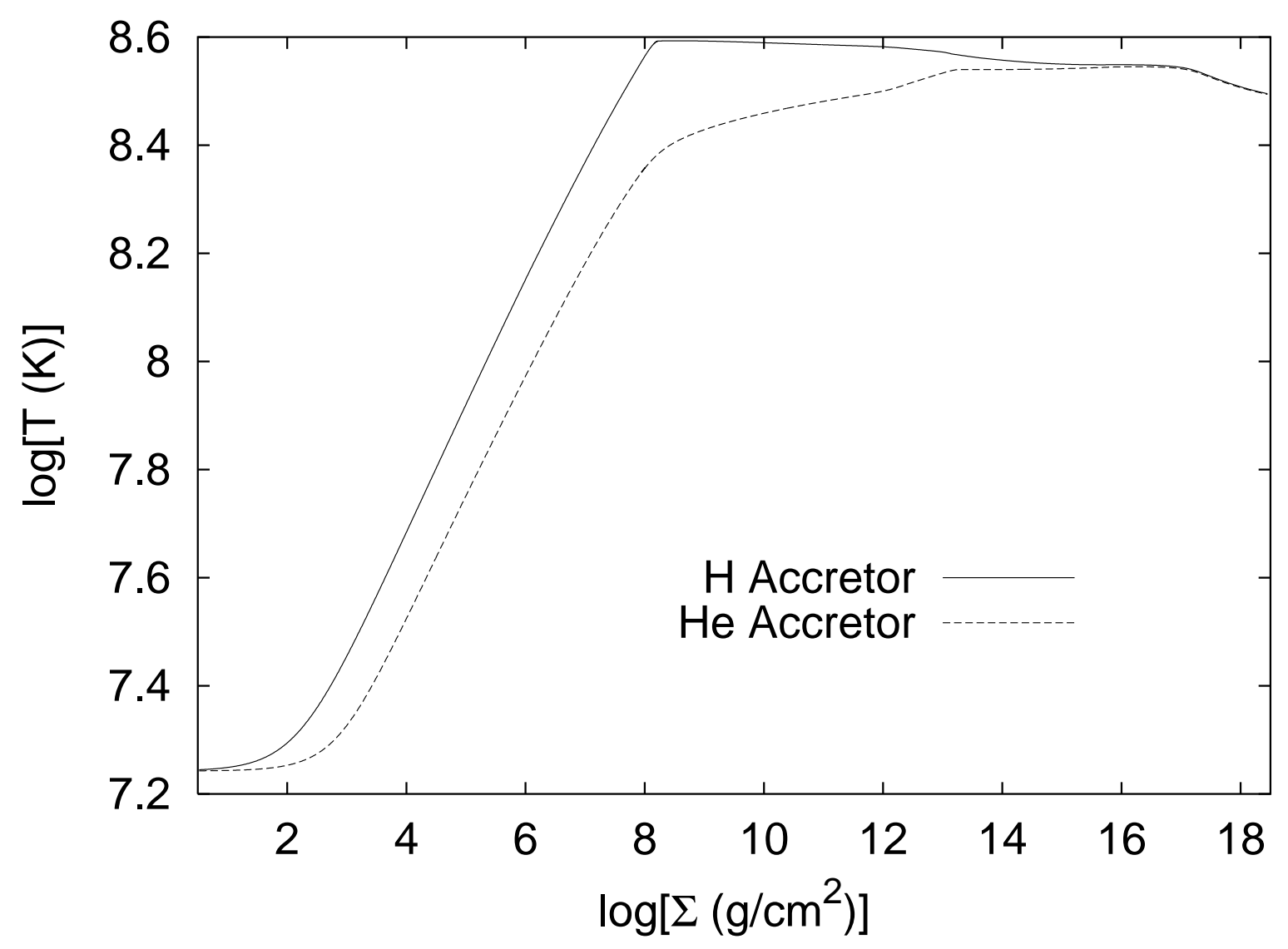

Fig. 3. - Thermal profiles for two neutron stars with different accreted gas compositions just before a superburst is triggered. The normalized accretion rate $l_{\text {acc }}=0.3$ for this and all subsequent thermal profiles. The mass fractions of the accreted gas are $X=0.7, Y=0.28$, $Z=0.004$, and $Z_{\mathrm{CNO}}=0.016$ for "H Accretor," and $X=0.1, Y=0.88, Z=0.004$, and $Z_{\mathrm{CNO}}=0.016$ for "He Accretor." Hydrogen burning releases much more energy per gram of fuel than helium burning. Consequently, the temperature of the superburst ignition region, $10^{11} \mathrm{~g} \mathrm{~cm}^{-2} \lesssim \Sigma \lesssim 10^{14} \mathrm{~g} \mathrm{~cm}^{-2}$, is greater for hydrogen accretors than for helium accretors. 


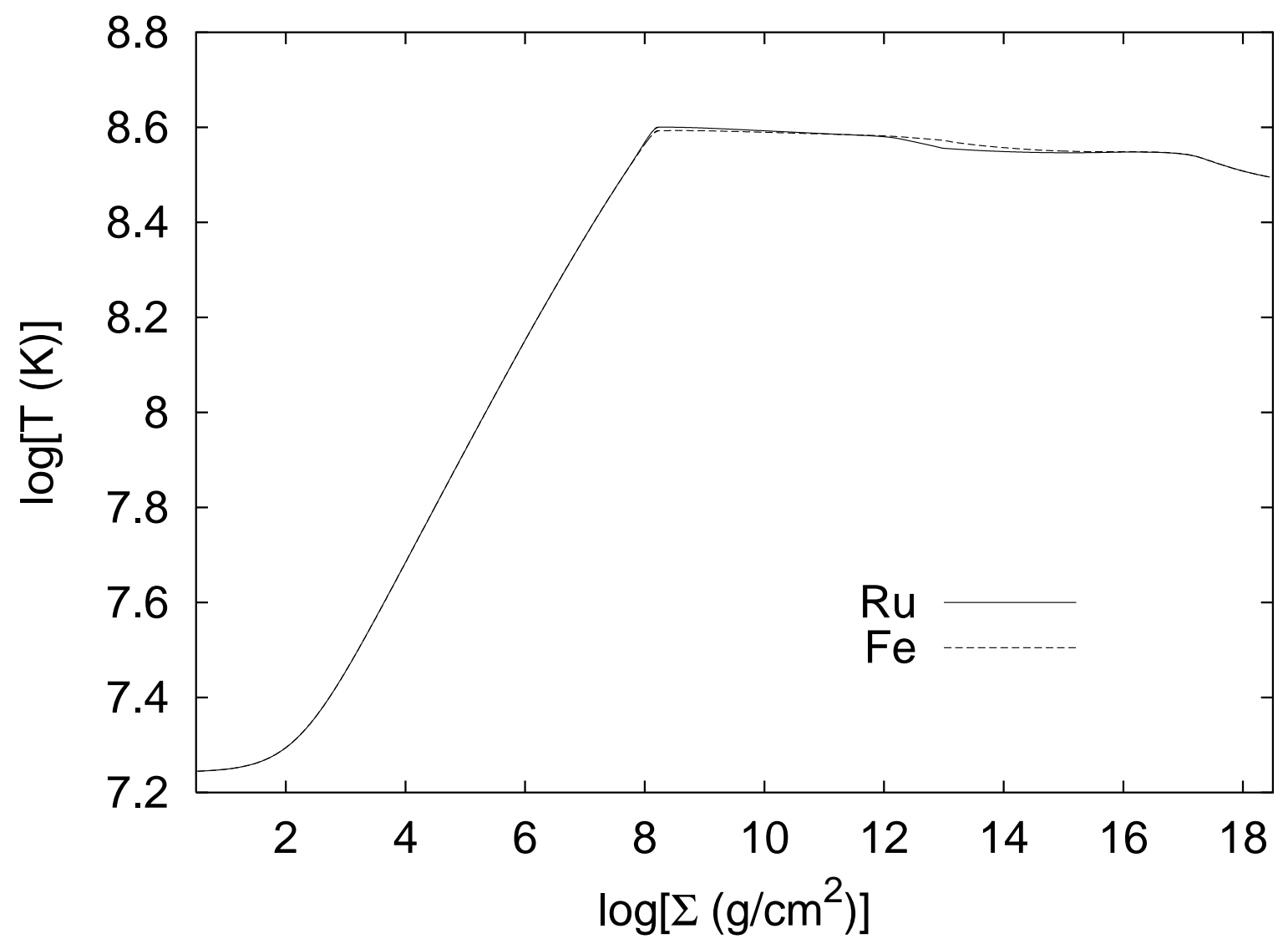

Fig. 4.- Thermal profiles for two neutron stars with different heavy element compositions in the accreted layer resulting from stable and unstable hydrogen and helium burning just before a superburst is triggered. "Ru" signifies a composition consisting of $30 \%{ }^{12} \mathrm{C}$ and $70 \%{ }^{104} \mathrm{Ru}$ by mass, and "Fe" signifies a composition consisting of $30 \%{ }^{12} \mathrm{C}$ and $70 \%{ }^{56} \mathrm{Fe}$ by mass. The heavy element composition has almost no effect on the thermal profile of the neutron star crust. 

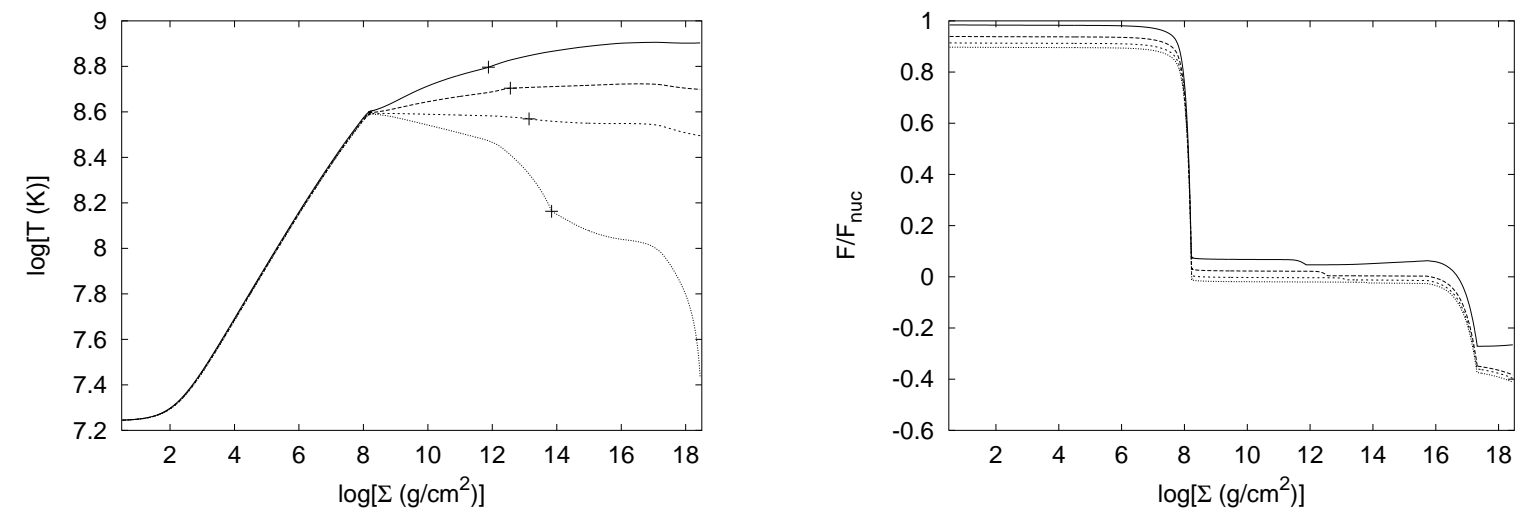

Fig. 5.- Thermal and net energy flux profiles of the crust for neutron stars with different core temperatures just before a superburst is triggered. Note that a positive value of $F$ denotes an outward flux. The crosses indicate the column depth at which a superburst is triggered. The core temperature significantly affects the thermal profile of the superburst ignition region, $10^{11} \mathrm{~g} \mathrm{~cm}^{-2} \lesssim \Sigma \lesssim 10^{14} \mathrm{~g} \mathrm{~cm}^{-2}$. The flux near this region is sensitive to several parameters including the core temperature, and it can be either positive or negative. 


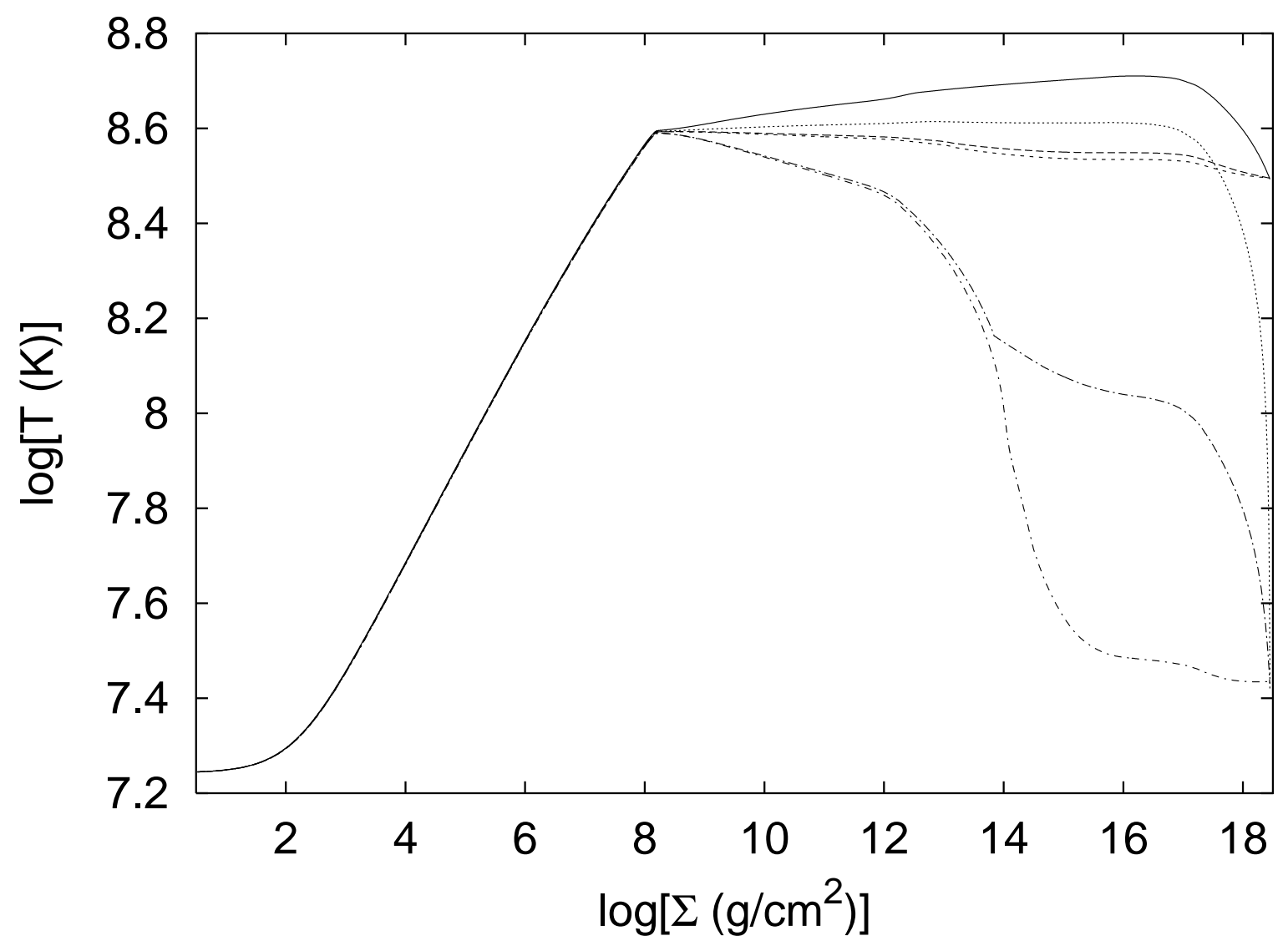

Fig. 6. - Thermal profiles for accreting neutron stars with different values of the impurity parameter in the substrate, below the accreted layer. We plot three thermal profiles for a neutron star with a core that emits neutrinos via modified URCA reactions (curves with $\log T \sim 8.5$ at $\log \Sigma \sim 18$ ) and three profiles for a star with a core that emits neutrinos via pionic reactions (curves with $\log T \sim 7.4$ at $\log \Sigma \sim 18$ ). For each star with a given core cooling mechanism, the three profiles correspond to impurity parameter values $Q=100$, 5.2 , and 0 from hottest to coldest. Note that impurity scattering has a significant effect on the thermal profile of the crust, especially in neutron stars with cold cores for which phonon scattering is weak. 


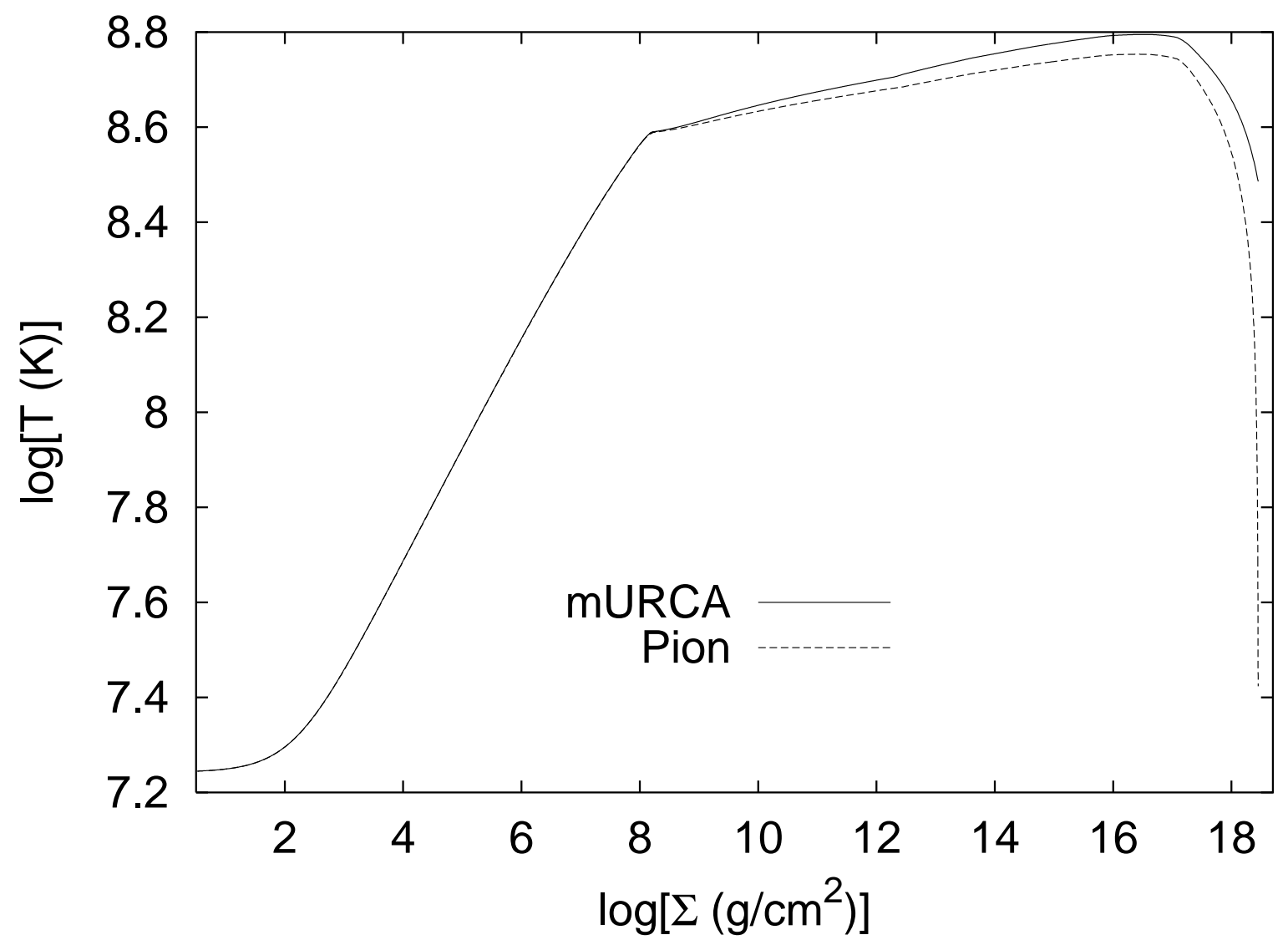

Fig. 7.- Thermal profiles for accreting neutron stars with disordered crusts. "mURCA" refers to a neutron star with a nonsuperfluid core that emits neutrinos via modified URCA reactions, and "Pion" refers to a neutron star with a core that emits neutrinos via pionic reactions. The low thermal conductivity of the disordered crust makes the crust rather insensitive to the core temperature. Contrast these results with those shown in Fig. 5. 

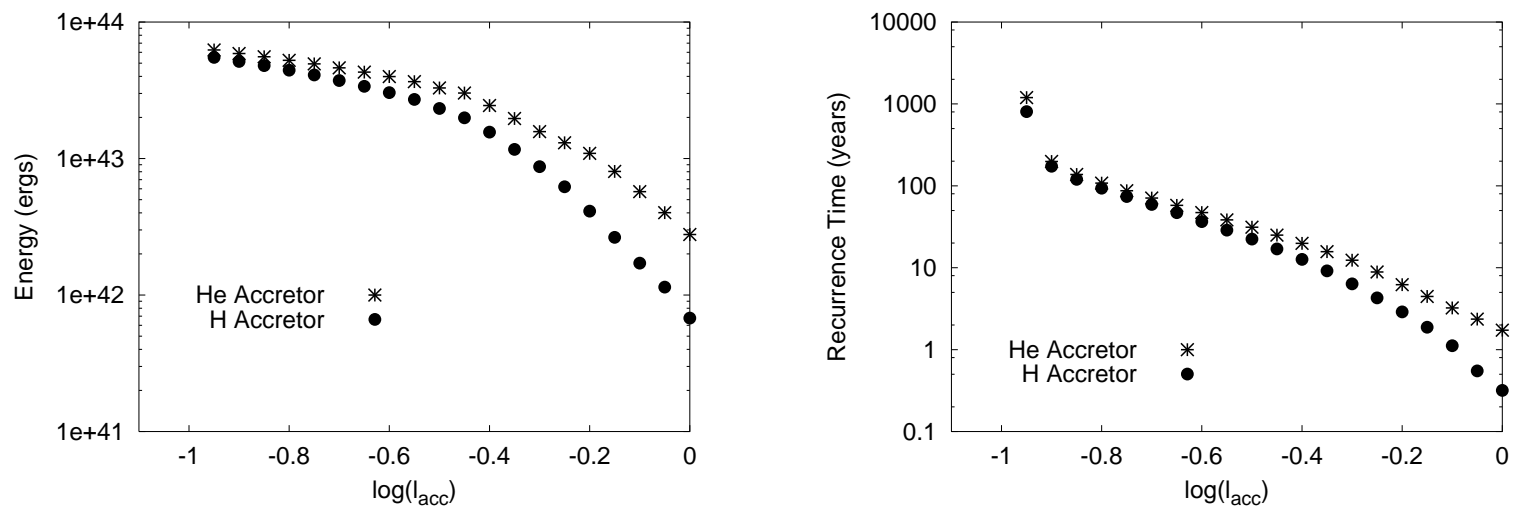

Fig. 8.- Superburst energies and recurrence times for mixed hydrogen/helium accretors and for helium accretors as a function of accretion rate. The core is assumed to consist of normal (nonsuperfluid) matter and to emit neutrinos via modified URCA reactions. The composition of the burned hydrogen and helium is taken to be $30 \%{ }^{12} \mathrm{C}$ and $70 \%{ }^{56} \mathrm{Fe}$ by mass (i.e. $C_{\mathrm{f}}=0.3$ ). We assume the fiducial value $C_{\mathrm{f}}=0.3$ for all calculations unless notified otherwise.
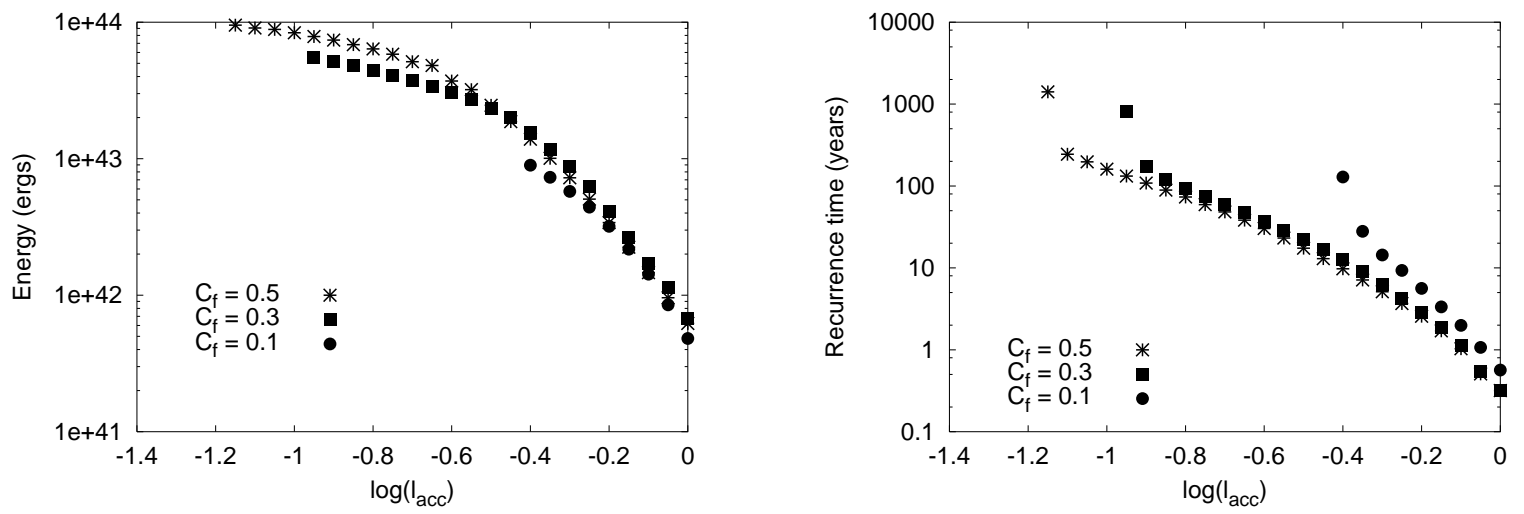

Fig. 9.- Superburst energies and recurrence times as a function of accretion rate for different values of $C_{\mathrm{f}}$. The core is assumed to emit neutrinos via modified URCA reactions. 

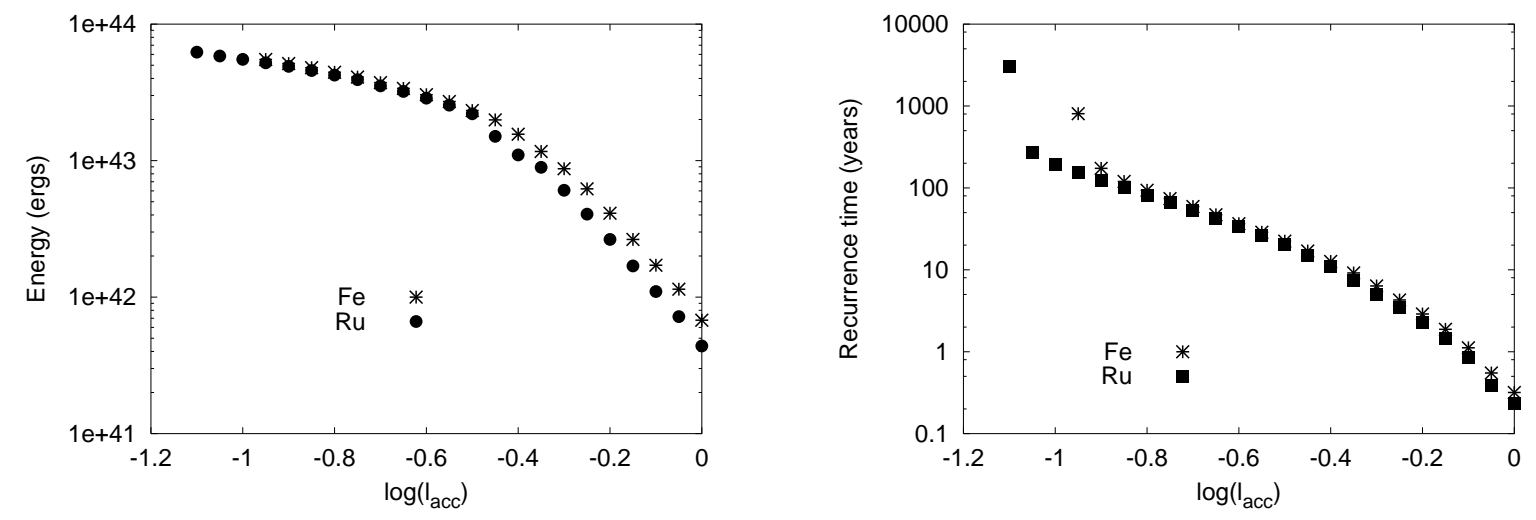

Fig. 10.- Superburst energies and recurrence times for different heavy element compositions in the neutron star crust as a function of accretion rate. The core is assumed to emit neutrinos via modified URCA reactions.
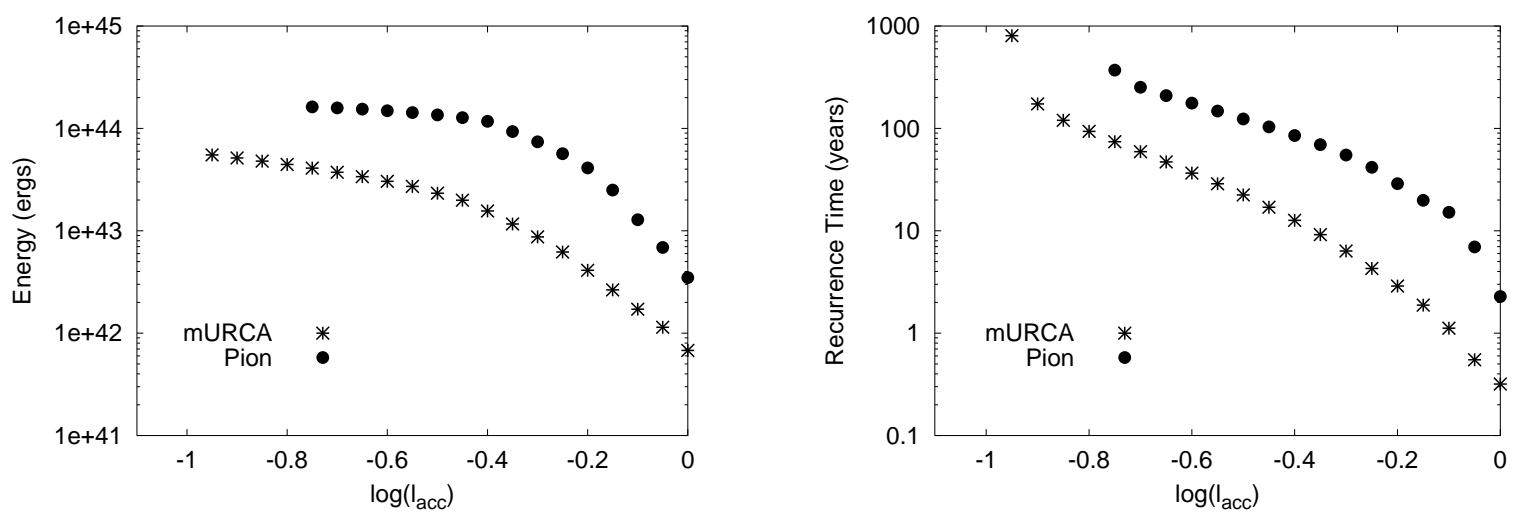

Fig. 11. - Superburst energies and recurrence times for two core neutrino cooling mechanisms as a function of accretion rate. The modified URCA and pion cooling models both assume a nonsuperfluid core. 

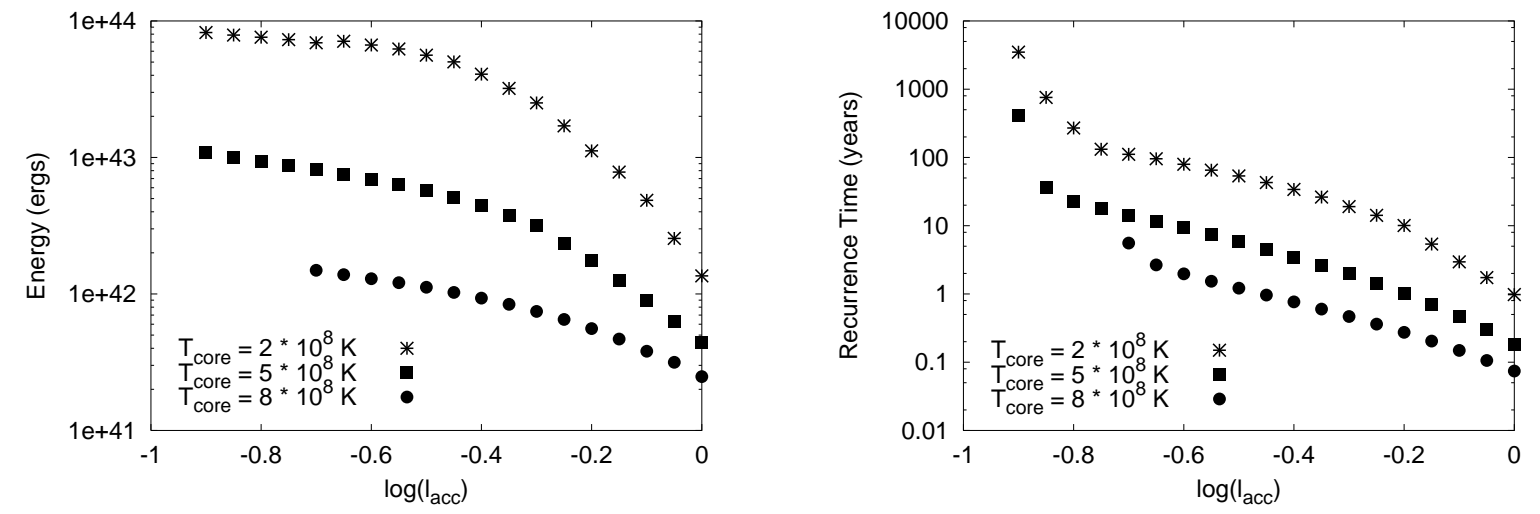

Fig. 12.- Superburst energies and recurrence times for different fixed temperatures at the crust-core interface as a function of accretion rate.
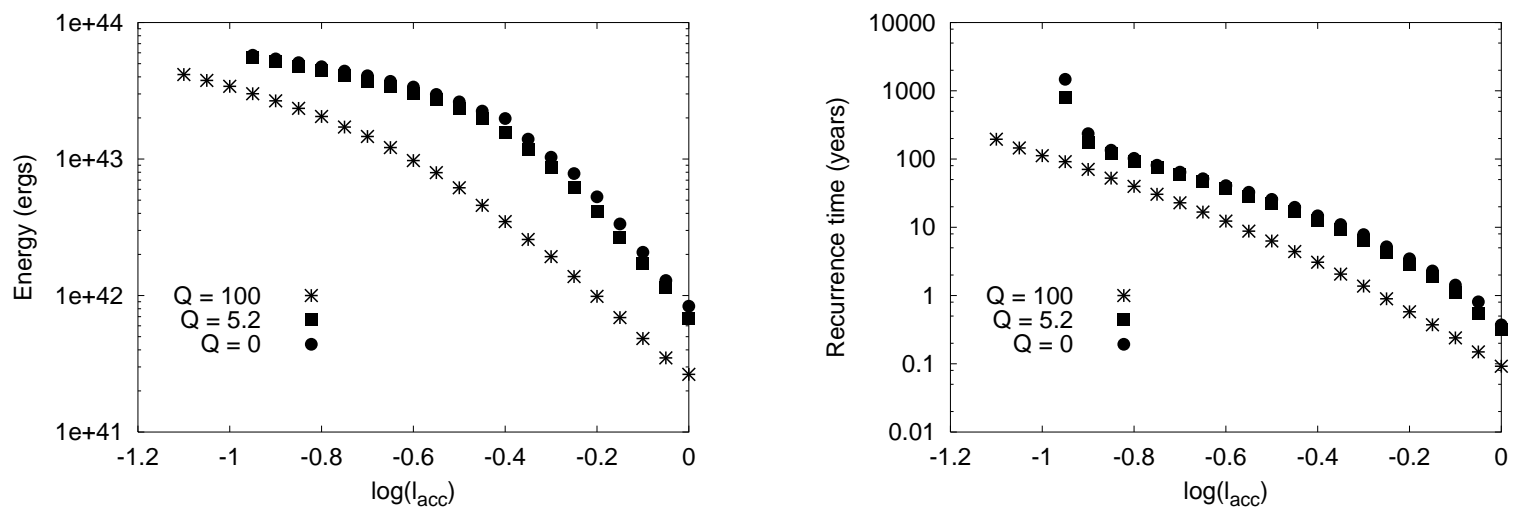

Fig. 13.- Superburst energies and recurrence times for neutron stars with different impurity parameter values in the substrate, below the accreted layer. The core is assumed to emit neutrinos via modified URCA reactions. 

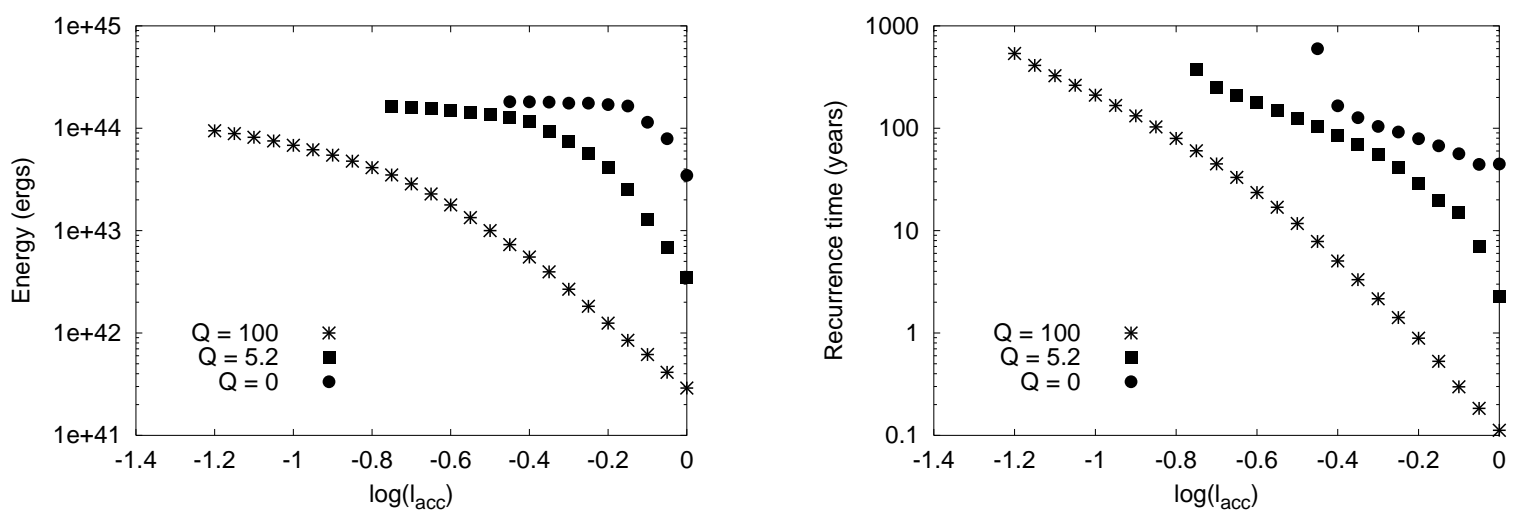

Fig. 14. - Superburst energies and recurrence times for neutron stars with different impurity parameter values in the substrate, below the accreted layer. The core is assumed to emit neutrinos via pionic reactions.
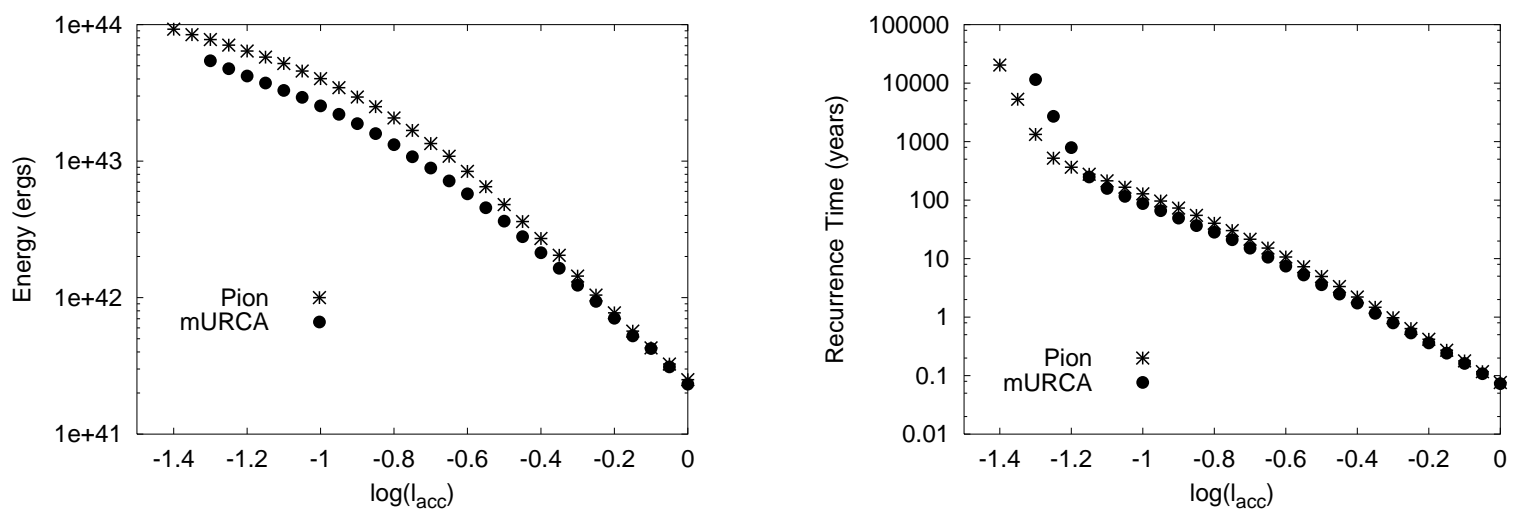

Fig. 15. - Superburst energies and recurrence times for neutron stars with completely disordered crusts as a function of accretion rate. 

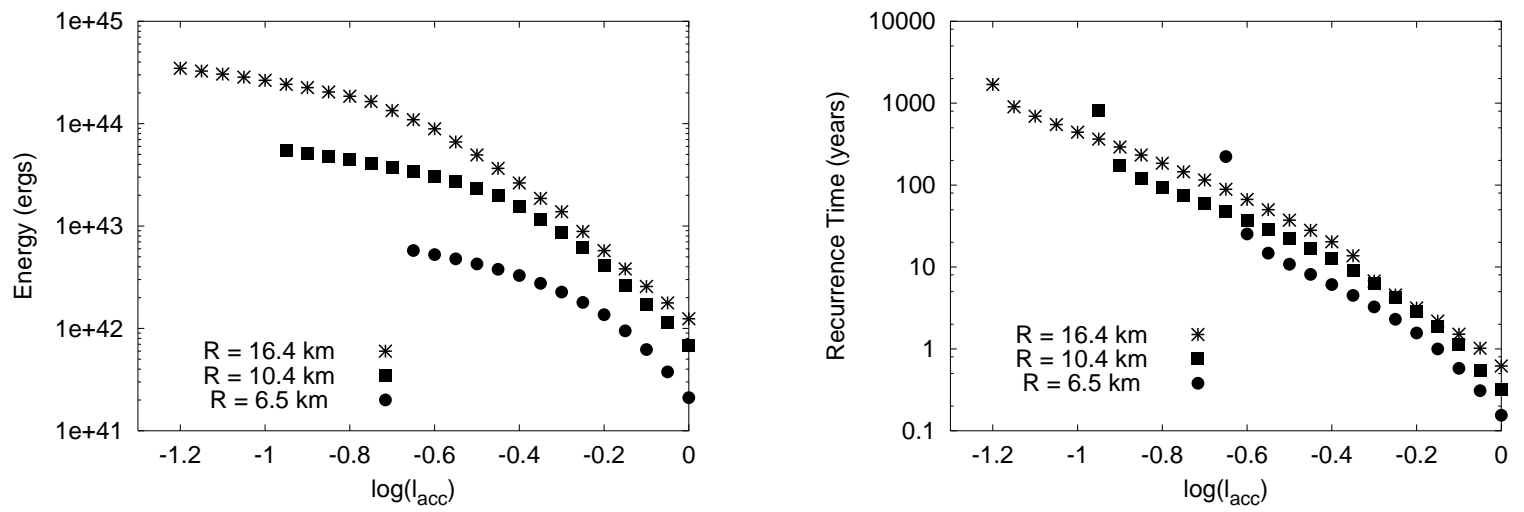

Fig. 16.- Superburst energies and recurrence times for different stellar radii as a function of accretion rate. The core is assumed to emit neutrinos via modified URCA reactions.
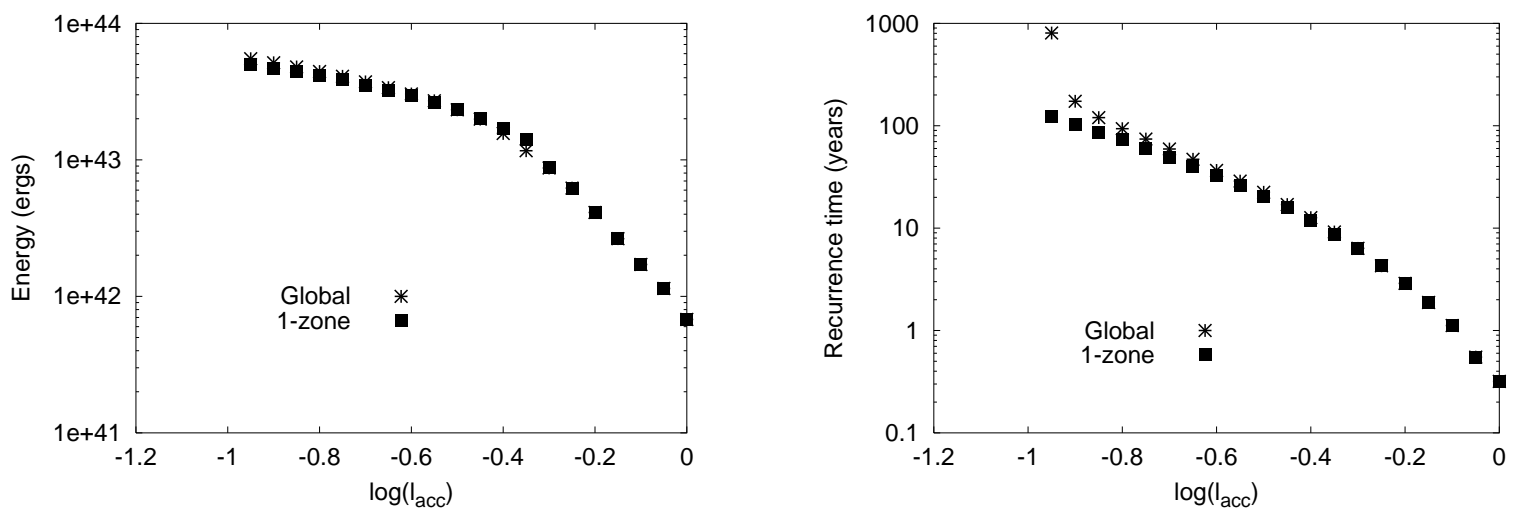

Fig. 17.- A comparison of superburst energies and recurrence times between our global linear stability analysis and the local one-zone approximation. "Global" signifies the results using our global linear stability analysis and "1-zone" signifies the results using the onezone approximation for which we set $\mathrm{d} \ln \epsilon_{\mathrm{C}} / \mathrm{d} \ln T=26$. The results for which we calculate $\mathrm{d} \ln \epsilon_{\mathrm{C}} / \mathrm{d} \ln T$ self-consistently are nearly identical. The core is assumed to emit neutrinos via modified URCA reactions. 

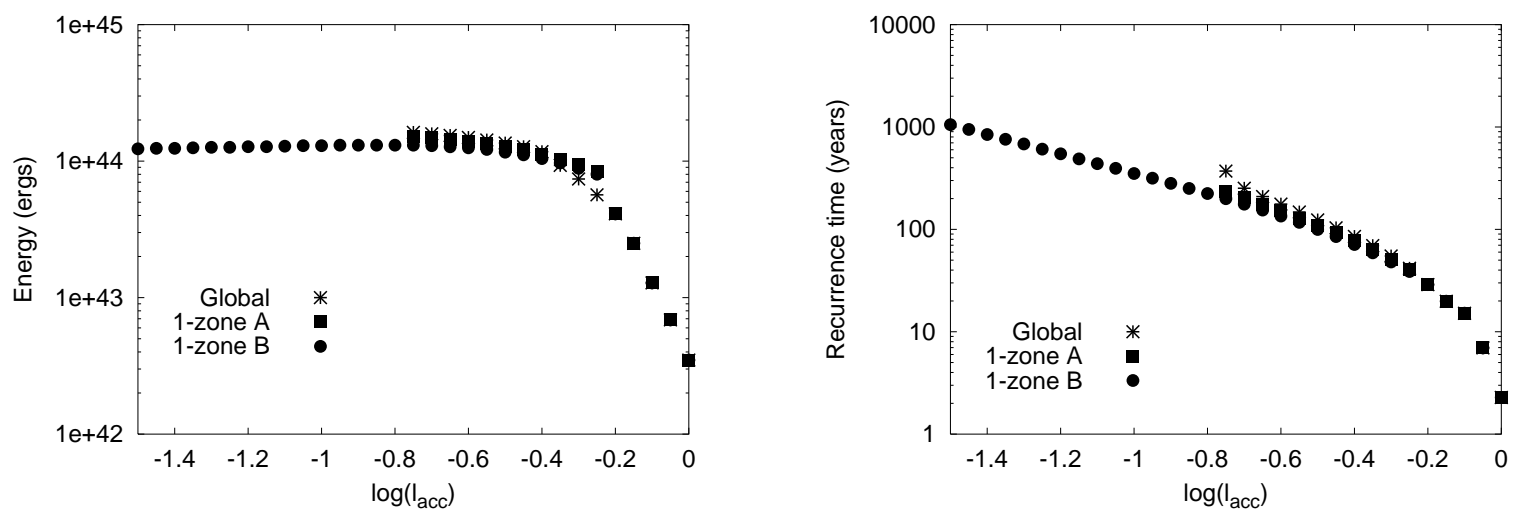

Fig. 18. - A comparison of superburst energies and recurrence times between our global linear stability analysis and the local one-zone approximation. "Global" signifies the results using our global linear stability analysis, "1-zone A" signifies the results using the onezone approximation for which we calculate $\mathrm{d} \ln \epsilon_{\mathrm{C}} / \mathrm{d} \ln T$ self-consistently (criterion $\mathrm{i}$ in $\S 6$ ), and "1-zone B" signifies the results using the one-zone approximation for which we set $\mathrm{d} \ln \epsilon_{\mathrm{C}} / \mathrm{d} \ln T=26$ (criterion iii). The core is assumed to emit neutrinos via pionic reactions. 